OPEN ACCESS

Edited by:

Silvia Masciarelli,

Catholic University of the Sacred

Heart, Italy

Reviewed by:

Sandeep K. Sharma,

Indian Institute of Toxicology Research

(CSIR), India

Rahul S. Samant

Babraham Institute (BBSRC),

United Kingdom

${ }^{*}$ Correspondence:

Ulrike Top

utopf@ibb.waw.pl

${ }^{\text {t}}$ These authors have contributed equally to this work

Specialty section:

This article was submitted to

Signaling,

a section of the journal

Frontiers in Cell and Developmental

Biology

Received: 16 November 2021

Accepted: 29 December 2021

Published: 17 January 2022

Citation:

Tahmaz I, Shahmoradi Ghahe S and Topf $U$ (2022) Prefoldin Function in Cellular Protein Homeostasis and Human Diseases.

Front. Cell Dev. Biol. 9:816214. doi: 10.3389/fcell.2021.816214

\section{Prefoldin Function in Cellular Protein Homeostasis and Human Diseases}

\author{
Ismail Tahmaz ${ }^{\dagger}$, Somayeh Shahmoradi Ghahe ${ }^{\dagger}$ and Ulrike Topf ${ }^{*}$ \\ Laboratory of Molecular Basis of Aging and Rejuvenation, Institute of Biochemistry and Biophysics, Polish Academy of Sciences, \\ Warsaw, Poland
}

Cellular functions are largely performed by proteins. Defects in the production, folding, or removal of proteins from the cell lead to perturbations in cellular functions that can result in pathological conditions for the organism. In cells, molecular chaperones are part of a network of surveillance mechanisms that maintains a functional proteome. Chaperones are involved in the folding of newly synthesized polypeptides and assist in refolding misfolded proteins and guiding proteins for degradation. The present review focuses on the molecular co-chaperone prefoldin. Its canonical function in eukaryotes involves the transfer of newly synthesized polypeptides of cytoskeletal proteins to the tailless complex polypeptide 1 ring complex (TRiC/CCT) chaperonin which assists folding of the polypeptide chain in an energy-dependent manner. The canonical function of prefoldin is well established, but recent research suggests its broader function in the maintenance of protein homeostasis under physiological and pathological conditions. Interestingly, non-canonical functions were identified for the prefoldin complex and also for its individual subunits. We discuss the latest findings on the prefoldin complex and its subunits in the regulation of transcription and proteasome-dependent protein degradation and its role in neurological diseases, cancer, viral infections and rare anomalies.

Keywords: prefoldin, molecular chaperone, proteostasis, TRIC, PFDN, neurodegenerative diseases, cancer

\section{INTRODUCTION}

Protein homeostasis (proteostasis) plays a vital role in various biological processes. Proteostasis is facilitated by the correct folding of proteins and removal of misfolded proteins to avoid protein aggregation, which can be toxic for the cell. To maintain proteostasis, cells contain various protein chaperones to check protein quality and conformation. A large family of chaperones, collectively called heat shock proteins (HSPs), are involved in the surveillance of misfolded and unfolded proteins in both the nucleus and cytosol (Hartl and Hayer-Hartl, 2002; Jones and Gardner, 2016). Non-native polypeptides must be sensed by chaperones and assisting proteins to assure correct folding. Disruption of the cooperation of chaperones and their assisting co-chaperones can result in protein misfolding and aggregation. Consequently, proteostasis can be impaired and drive the development of various pathologies (Hartl and Hayer-Hartl, 2002; Jones and Gardner, 2016).

Monomeric molecular chaperones are well established to assist in the folding of proteins into their native structure and responsible for the remodeling of proteins with erroneous conformations (Saibil, 2013). In addition to monomeric molecular chaperones, oligomeric chaperonins contribute to protein folding. Chaperonins can be divided into two groups. Group I chaperonins are found in bacteria and organelles of endosymbiotic origin (e.g., chloroplasts and mitochondria). Group II chaperonins exist in 

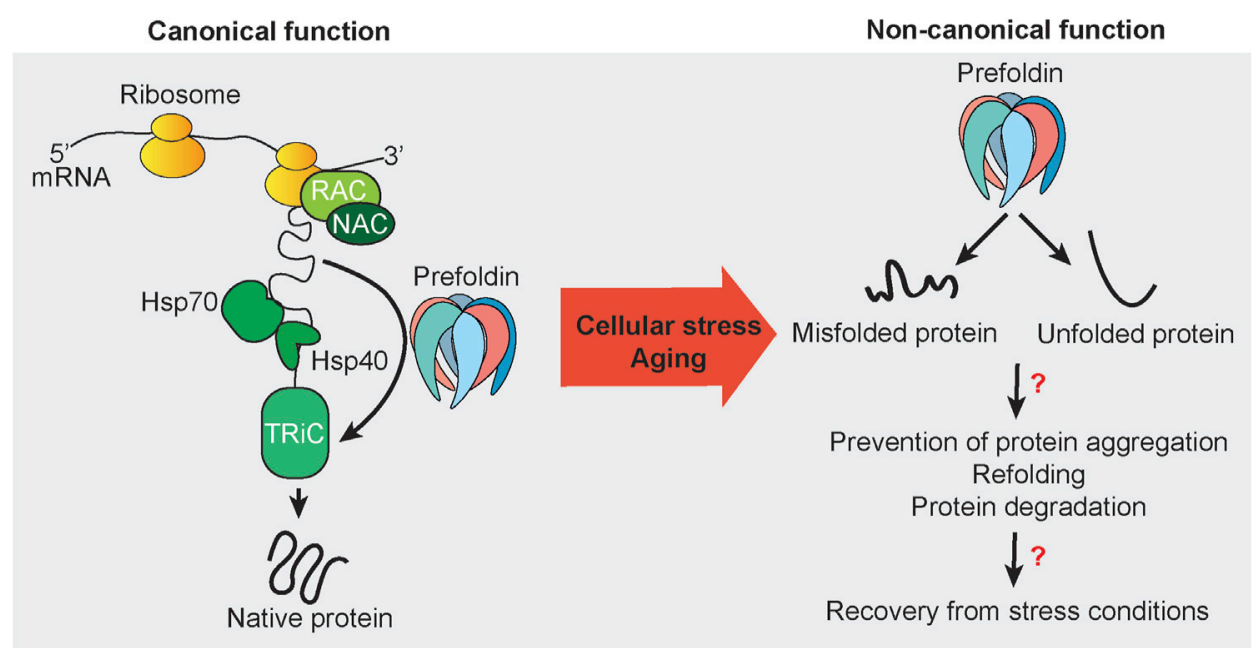

FIGURE 1 | Canonical and non-canonical functions of prefoldin. Nascent chains that emerge from actively translating ribosomes are subjected to protein folding that is mediated by the protein chaperones RAC, NAC, Hsp70, Hsp40, and TRiC. Some newly synthesized proteins utilize the co-chaperone prefoldin to guide substrates directly to its downstream folding chaperone TRiC. The canonical function of prefoldin results in substrate proteins with the native fold. Under stress conditions and potentially during aging, prefoldin is associated with misfolded and unfolded proteins. Mostly unknown are the nature of such cellular substrates in vivo and cellular consequences and mechanisms that may be necessary to recover from cellular stress conditions to restore cellular homeostasis.

eukaryotes and archaea (Russmann et al., 2012). One well-known member of Group II chaperonin is tailless complex polypeptide 1 ring complex (TRiC)/chaperonin containing tailless complex polypeptide 1 (CCT). TRiC/CCT localizes to both the nucleus and cytosol (Marracci et al., 2015).

Unlike prokaryotes, eukaryotes and archaea have another chaperone-assisted protein (Sahlan et al., 2018). Prefoldin is a cochaperone of TRiC/CCT (Millan-Zambrano and Chavez, 2014; Zhang J. et al., 2020). It is a heterohexameric protein complex (Glover and Clark, 2015) that is composed of two a subunits (PFD3 and PFD5) and four $\beta$ subunits [PFD1, PFD2, PFD4, and PFD6; (Siegert et al., 2000; Whitehead et al., 2007). All subunits differ in their amino acid sequence. In contrast, archaea prefoldin complex consists of six subunits composed of two identical $\alpha$ and four identical $\beta$ subunits (Sahlan et al., 2018). Although prefoldin was not found in prokaryotes, its genetic origin is similar to chaperones that were found in prokaryotes (Bogumil et al., 2014). Likewise, the prefoldin complex is evolutionarily conserved in higher eukaryotes, including humans and plants (Siegert et al., 2000; Cao, 2016).

The prefoldin complex assists in the folding of newly synthesized polypeptides that are produced on ribosomes (Figure 1). In archaea, prefoldin exerts this function in combination with group II chaperonin. In eukaryotes, the canonical function of prefoldin is thought to be more specialized. In eukaryotes, prefoldin transfers mainly the cytoskeletal proteins actin and tubulin to TRiC/CCT (Abe et al., 2013; Povarova et al., 2014; Zhang et al., 2016). This transfer is also supported by cooperation with the monomeric chaperones HSP40 and HSP70 in a co-translational manner (MillanZambrano and Chavez, 2014; Gestaut et al., 2019) and required for accelerating the maturation of cytoskeletal proteins (Gestaut et al., 2019). During this process, nascent polypeptide chains bind to prefoldin in the initial phase (Kabir et al., 2011) and are transferred to TRiC/CCT in late stages of protein folding (Comyn et al., 2016; Gestaut et al., 2019). Binding of prefoldin subunits with TRiC/CCT subunits decreases the association of nascent chain with prefoldin (Gestaut et al., 2019; Liang et al., 2020). Thus, nascent chains are released from prefoldin and folded in an adenosine triphosphate (ATP)-dependent manner by TRiC/CCT. The holdase function of prefoldin is ATP-independent, similar to stress-inducible small HSPs (Webster et al., 2019). Its ATP independence raises the question of whether prefoldin has additional functions under conditions of low cellular energy levels such as mitochondrial dysfunction and metabolic disorders, aging, or chronic stress (Chaudhari and Kipreos, 2018; Andreasson et al., 2019; Fang et al., 2021).

Actin comprises $5-10 \%$ of cellular protein mass (Cooper, 2000). Thus, prefoldin is thought to be abundant with constitutive expression. However, the regulation of prefoldin expression has been scarcely addressed in the literature. The turnover of prefoldin subunits is regulated by ubiquitin-dependent degradation. However, prefoldin can become resistant to ubiquitination by forming a $\beta$-hairpin and coil-coil structure (Liang et al., 2020). Since the discovery of prefoldin in 1998 (Vainberg et al., 1998), much research has been performed to elucidate the prefoldin structure (Hansen et al., 1999) and describe its canonical functions as a co-chaperone (Sahlan et al., 2018). However, recent research has indicated involvement of the prefoldin complex and its subunits in withstanding cellular stress and maintaining protein homeostasis. Research on the non-canonical function of prefoldin is still scattered, consisting of the regulation of transcription, the regulation of protein aggregate formation, and protein degradation (Figure 1).

Alongside fundamental research describing various functions of prefoldin it is suggested that prefoldin is a potential biomarker and therapeutic target for human diseases that are associated with the disruption of protein homeostasis. The present review discusses non- 
TABLE 1 | Interactions between prefoldin subunits and other proteins.

\begin{tabular}{|c|c|c|c|c|}
\hline Prefoldin subunit & Interactor & Organism & Function & References \\
\hline \multirow[t]{4}{*}{ PFDN1 } & HLA-G & Human & Progression of pregnancy & Liu et al. (2013) \\
\hline & cyclin A & Human & Cancer progression & Wang et al. (2017) \\
\hline & RABV & Mouse & Upregulation in virus infection & Zhang et al. (2015) \\
\hline & FILIP1L & Mouse and human & Progression of mucinous colon cancer & Kwon et al. (2021) \\
\hline \multirow[t]{3}{*}{ PFD2/PFDN2 } & Irc15 & Yeast & Sister chromatid cohesion & Ming Sun et al. (2020) \\
\hline & $\gamma$-synuclein & Mouse & Expression regulation in neurodegenerative disease & Chintalapudi and Jablonski, (2017) \\
\hline & DELLA & Populus & Alteration of lignin content & Zhang W et al., (2020) \\
\hline \multirow[t]{4}{*}{ PFD3 } & $\mathrm{pVHL}$ & Human & Tumor suppression & Kim et al. (2018) \\
\hline & HDAC1 & Human & Delivery to CCT complex & Banks et al. (2018) \\
\hline & hMSH4 & Human & Degradation & Xu and Her, (2013) \\
\hline & DELLA & A. thaliana & Stress-dependent interaction & Locascio et al. (2013) \\
\hline \multirow[t]{2}{*}{ PFD4 } & $\operatorname{Irc15}$ & Yeast & Sister chromatid cohesion & Ming Sun et al. (2020) \\
\hline & LSM8 & A. thaliana & Splicing & Esteve-Bruna et al. (2020) \\
\hline \multirow[t]{12}{*}{ PFDN5 } & c-Myc & Human & Inhibitory effect on oncogenes & Satou et al. (2001) \\
\hline & $\mathrm{TIF} 1 \beta$ & Human & Inhibitory effect on oncogenes & Hagio et al. (2006) \\
\hline & Rabring7 & Human & c-Myc degradation & Narita et al. (2012) \\
\hline & Egr1 & Human & c-Myc inhibition & Yoshida et al. (2008) \\
\hline & ARFP/F & Human & c-Myc activation & Ma et al. (2008) \\
\hline & $\mathrm{p} 73 a$ & Human & c-Myc inhibition & Watanabe et al. (2002) \\
\hline & $\mathrm{p} 63 \alpha$ & Human & c-Myc activation & Han et al. (2016) \\
\hline & $\Delta \mathrm{Np63a}$ & Human & Senescence & Chen et al. (2018) \\
\hline & Fabp4 & Mouse & Regulation of sperm differentiation at transcription & Yamane et al. (2015) \\
\hline & PLa2g3 & Mouse & Transcriptional regulation of sperm number and motility & Yamane et al. (2015) \\
\hline & PLa2g10 & Mouse & Transcriptional regulation of sperm number and motility & Yamane et al. (2015) \\
\hline & DELLA & A. thaliana & Stress-dependent interaction & Locascio et al. (2013) \\
\hline \multirow[t]{3}{*}{ PFD6/PFD-6 } & DELLA & A. thaliana & Stress-dependent interaction & Locascio et al. (2013) \\
\hline & FOXO & C. elegans & Transcriptional regulation of longevity & Son et al. (2018) \\
\hline & Irc15 & Yeast & Sister chromatid cohesion & Ming Sun et al. (2020) \\
\hline All subunits & mst & Drosophila & Assembly of spindle microtubules & Palumbo et al. (2015) \\
\hline
\end{tabular}

canonical functions of prefoldin in eukaryotes and the involvement of prefoldin in human diseases.

\section{NON-CANONICAL FUNCTIONS OF THE PREFOLDIN COMPLEX}

Apart from the canonical function of the cytosolic prefoldin complex as a co-chaperone for the co-translational folding of cytoskeletal proteins, all prefoldin subunits were found to have unique and specific interactors. These interactions presumably allow prefoldin subunits to engage in non-canonical cellular functions (Simons et al., 2004), but describing all of these interactions is beyond the scope of this review. Table $\mathbf{1}$ summarizes the identified interactors with different prefoldin subunits. A recent review described links between interactors of prefoldin subunits and the progression of cancer (Liang et al., 2020; Herranz-Montoya et al., 2021). The sections below focus on the involvement of prefoldin subunits in transcription, protein aggregation, and degradation.

\section{Regulation of Transcription}

Prefoldin was described to be a cytoplasmic protein complex (Geissler et al., 1998). The deletion of PFD1 in yeast resulted in unexpected defects in transcription elongation (MillanZambrano et al., 2013), but these findings complemented other studies and suggested a role for prefoldin in the nucleus (Millan-Zambrano and Chavez, 2014; Mousnier et al., 2007; Watanabe et al., 2002) (Figure 2). All prefoldin subunits were found to exhibit nucleo-cytoplasmic localization in the yeast Saccharomyces cerevisiae (Millan-Zambrano et al., 2013). This newly identified transcriptional function of prefoldin subunits was independent of actin assembly in the cytoplasm and independent of the involvement of nuclear actin in transcription elongation. The deletion of PFD1 decreased the RNA polymerase II occupancy of gene bodies (Figure 2A). This was explained by the role of prefoldin in the removal of histones from chromatin. These data were supported by numerous genetic interactions between prefoldin subunits and chromatin factors (Millan-Zambrano et al., 2013; Ming Sun et al., 2020). Genetic interactions between prefoldin and TRiC/CCT components and chromatin factors were similar and suggested a combined action of chaperonin and its co-chaperone in transcription regulation in the nucleus. Furthermore, the physical interaction between histone deacetylase 1 (HDAC1) and prefoldin and TRiC/CCT suggested the requirement for chaperone-mediated folding before its assembly into the active complex (Banks et al., 2018) (Figure 2B). This further strengthened the involvement of 


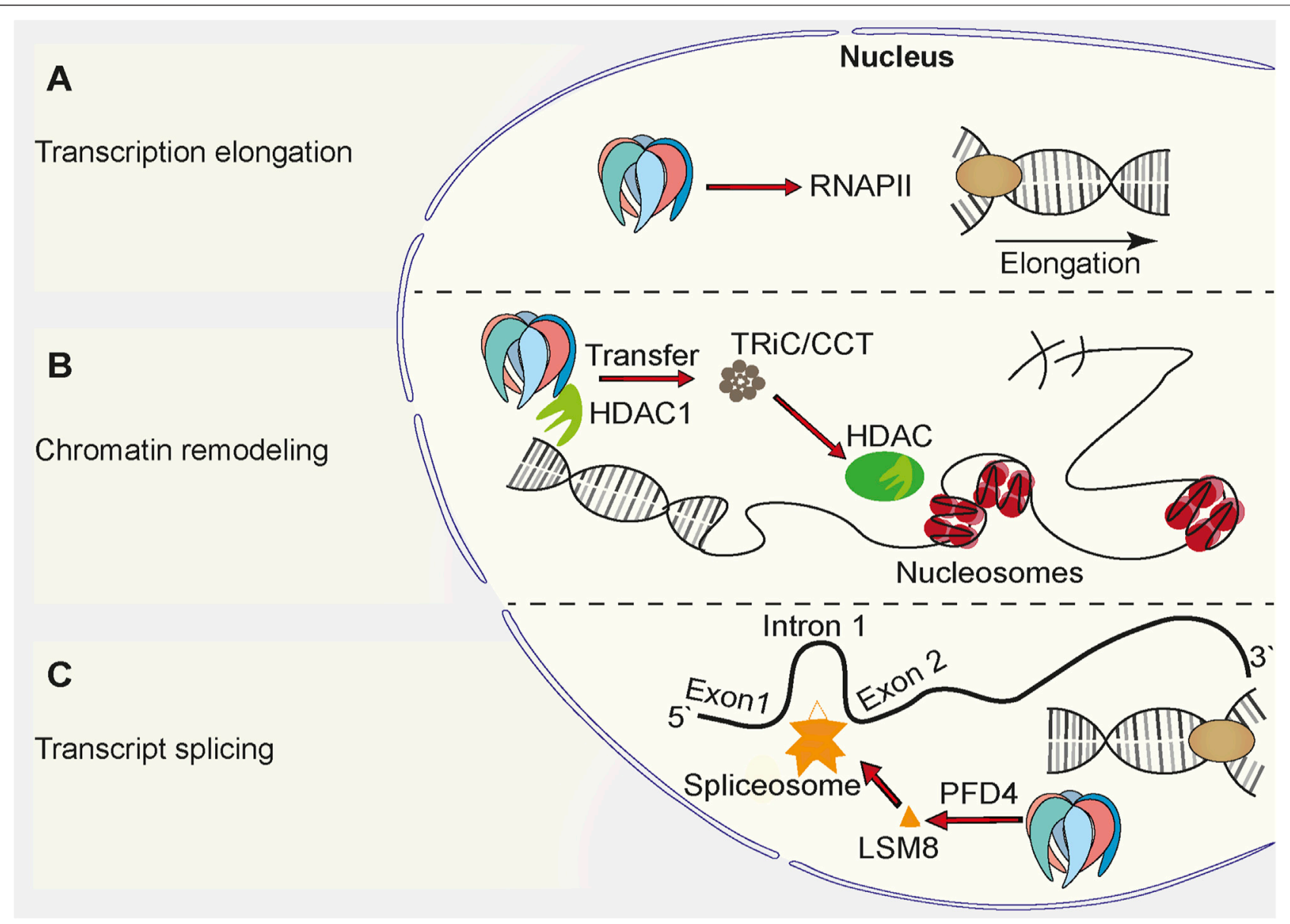

FIGURE 2 | Function of prefoldin complex in the cell nucleus. (A) Prefoldin stimulates transcription elongation by promoting binding of RNA Polymerase II (RNAPII) to euchromatin. (B) Prefoldin interacts with HDAC1 and supports incorporation of it into its active complex via TRiC/CCT complex. The interaction favors chromatin remodeling. (C) The PFD4 subunit is required for the incorporation of the spliceosome core complex subunit, LSM8, into the spliceosome.

prefoldin in the remodeling of chromatin and transcription regulation. A recent study in Arabidopsis showed that PFD4 mediates the chaperone-client interaction between Hsp90 and LSM8, a central component of the spliceosome core complex. Prefoldin was shown to be important for maintaining adequate levels of the spliceosome core complex (Esteve-Bruna et al., 2020) (Figure 2C). A strong genetic interaction between $p f d 4 \Delta$ (gim3 in yeast) and lsm8 in yeast (Costanzo et al., 2016) prompted EsteveBruna et al to the notion that the functional interaction between prefoldin and the spliceosome could be conserved. The implication of prefoldin in the regulation of co-translational splicing effects was also recently demonstrated for human prefoldin (Payán-Bravo et al., 2021). The binding of prefoldin to transcribed genes was detected genome-wide. The loss of prefoldin function led to a decrease in the phosphorylation of elongating polymerase II and decrease in the binding of splicing factors to chromatin. Links between prefoldin and cellular splicing machinery thus add another level of complexity to the ways in which prefoldin influences transcriptional processes in the nucleus.

\section{Role in Cellular Stress Defense}

The importance of prefoldin-dependent regulation of gene expression became eminent under cellular stress conditions. The green fluorescent protein-tagged Pfd1 exhibited an increase in nuclear staining when cells were shifted to a restrictive growth temperature (Millan-Zambrano et al., 2013). This finding in yeast was consistent with studies in plants that investigated the regulated migration of prefoldin from the cytoplasm to the nucleus (Locascio et al., 2013). Here, the shuttling of plant prefoldin complex depended on the interaction of PFD3 and PFD5 with the transcriptional regulator DELLA under conditions of environmental and endogenous cue exposure (Figure 3A). A mutant of Arabidopsis that harbored deletions of all six prefoldin subunits was viable, and loss of the prefoldin complex did not significantly alter plant development under normal growth conditions. The prefoldin complex knockout mutant performed better than the wild type under osmotic stress conditions (Blanco-Touriñán et al., 2021). This indicates that 


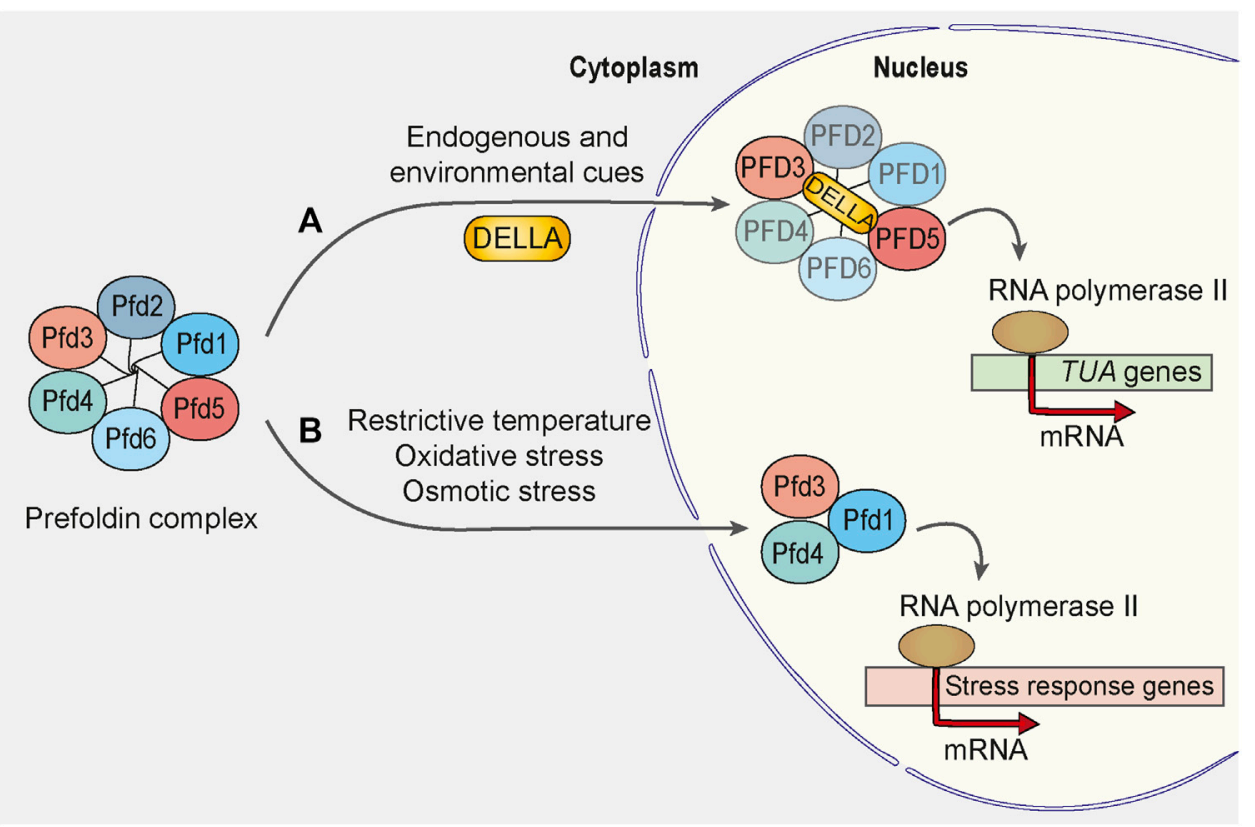

FIGURE 3 | Prefoldin complex is involved in transcriptional regulation upon cellular stress. (A) In Arabidopsis, endogenous and environmental stimulators control the growth rate of a plant by inducing the DELLA-mediated accumulation of prefoldin complex in the cell nucleus. PFD3 and PFD5 interact directly with DELLA, but integrity of the prefoldin complex during the translocation remains unaffected. In the nucleus, prefoldin complex or some of its subunits activate the transcription of $a-$ tubulin encoding genes (TUA). (B) In response to exposure of yeast Saccharomyces cerevisiae to stress stimuli such as restrictive temperature, oxidative and osmotic stress, Pfd1, Pfd3 and Pfd4 subunits localize to the nucleus to facilitate the transcription elongation of some stress-response genes. Pfd1, Pfd3 and Pfd4 may form a distinct complex from the hexameric prefoldin complex because the absence of other prefoldin subunits and consequent disruption of the prefoldin complex does not suppress the stress response in S. cerevisiae.

prefoldin is needed to respond to stress conditions and/or environmental changes. Consistent with recent findings in plants are findings in yeast, in which Pfd3 (also called Gim2 or Pac10), Pfd4 (also called Gim3), and Pfd1 (also called Gim6) were shown to be involved in oxidative and osmotic stressactivated transcription (Amorim et al., 2017) (Figure 3B).

The deletion of prefoldin also affected yeast viability upon exposure to other exogenous stressors. A genome-wide screen of haploid deletion mutants found that subunits of the prefoldin complex were among the most sensitive mutants to arsenite treatment (Pan et al., 2010). Exposure to high temperatures decreased the growth of a PFD5 deletion mutant in yeast (Gestaut et al., 2019). The induction of endoplasmic reticulum stress by thapsigargin treatment decreased cell viability in the absence of the prefoldin complex in mammalian cells (Abe et al., 2013). These stressors generally increase proteostatic stress in the cell, which might be an indication that prefoldin is required for the maintenance of protein homeostasis under conditions of high protein burden.

\section{Influence on Protein Turnover and Quality Control}

Protein turnover defines the balance between protein production and protein degradation. Importantly, the correct folding of newly synthesized polypeptides is a limiting factor of both fundamental cellular processes (Rothman, 2010). Defects in the folding process can stall translation and require an increase in the removal of unfolded or misfolded proteins from the cell (Goldman et al., 2015). Nascent chains that emerge from the actively translating ribosome are passed to chaperones of the ribosome-associated complex (RAC) and nascent polypeptide-associated complex (NAC), which assist the folding of $\sim 70 \%$ of polypeptides (Balchin et al., 2016). Downstream of ribosomes (i.e., the hub of the chaperone network), Hsp70, together with Hsp40s, folds $20 \%$ of the proteome (Balchin et al., 2016). Ten percent of these proteins are passed further downstream to multi-domain cylindrical complexes, group II chaperonins [TRiC/CCT; Hsp60 chaperones in archaea and the eukaryotic cytosol (Thulasiraman et al., 1999; Yam et al., 2008);]. Some proteins bypass Hsp70 and are directly transferred to chaperonins via the prefoldin chaperone complex (Balchin et al., 2016) (see also Figure 1). The prefoldin structure was determined in archaea, revealing a jellyfish-shaped complex where the six subunits form six long coiled coils that are attached to a base of $\beta$ barrels (Leroux et al., 1999; Fandrich et al., 2000; Siegert et al., 2000; Ohtaki et al., 2008). Biochemical experiments and low-resolution electron microscopy studies confirmed this structural arrangement for human prefoldin (Martin-Benito et al., 2002; Simons et al., 2004; Aikawa et al., 2015; Gestaut et al., 2019). In eukaryotes, charged residues in the distal end of coiled coils of certain prefoldin subunits bind to actin and tubulin, and defined residues in each of these substrates are necessary for this interaction. Thus, prefoldin 

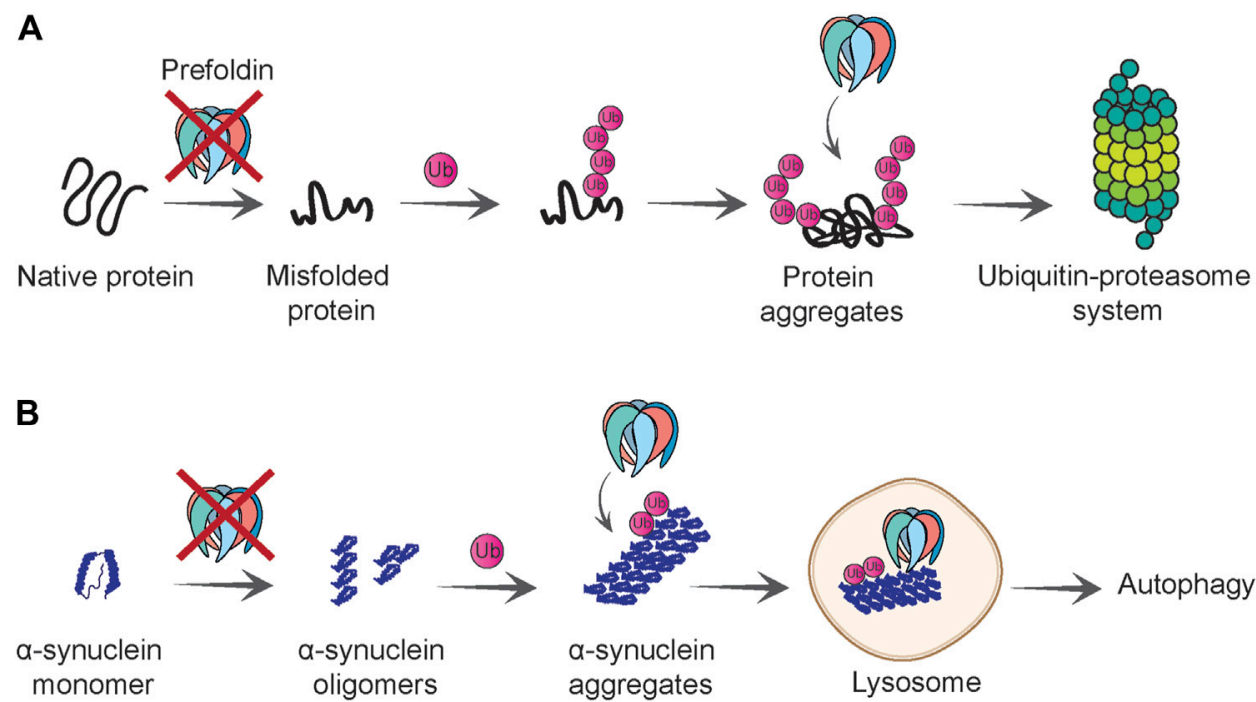

FIGURE 4 | Role of prefoldin complex in protein quality control and degradation. (A) Prefoldin is required for polypeptides to gain native fold and loss of prefoldin function causes protein misfolding. Misfolded proteins that cannot be refolded are polyubiquitinated. Prefoldin complex co-localizes with the polyubiquitinated proteins and presumably facilitates their degradation by the ubiquitin-proteasome system. (B) Prefoldin complex prevents aggregation of $\alpha$-synuclein at initial steps of oligomerization. If $a$-synuclein oligomers form aggregates, they will be ubiquitinated and in proximity to prefoldin complex delivered to the lysosome in which aggregates can be degraded in an autophagy-dependent manner.

binding is specific and may involve the combinatorial binding of different subunits for different targets. However, in contrast to archaeal prefoldin, which was shown to interact with numerous non-native substrates (Arranz et al., 2018), knowledge of the substrate landscape in eukaryotic prefoldin is limited. Interestingly, genome-wide genetic interaction screens in yeast revealed a large variety of genes that when deleted in the background of deletions of single prefoldin subunits result in a synthetic lethal phenotype (Goehring et al., 2003; Krogan et al., 2003; Tong et al., 2004; Friesen et al., 2006; Mitchell et al., 2008). This might indicate that prefoldin has a broader cellular function than previously anticipated.

A few findings suggest a link between the prefoldin complex and the removal of proteins that are targeted to the proteasome (Abe et al., 2013; Comyn et al., 2016) (Figure 4A). In a mammalian cell culture model, the prefoldin complex colocalized with ubiquitinated proteins that were found in aggregates upon proteasome inhibition. Intriguingly, lower levels of the prefoldin complex resulted in the higher accumulation of poly-ubiquitinated proteins that were resistant to detergent. This effect depended on concomitant inhibition of the proteasome. A decrease in prefoldin function that was associated with an insufficient protein degradation system most likely overwhelmed the cellular proteostasis system, consequently leading to an increase in cell death (Abe et al., 2013).

Overexpression of the prefoldin complex lowered levels of detergent-insoluble ubiquitinated protein species upon proteasome inhibition, but this conferred only a minimal beneficial effect on cell viability. A mouse model that expressed PFDN5 with a point mutation (MM-1a/PFDN5) showed normal PFDN5 expression and no detectable differences in expression of the prefoldin complex (Lee et al.,
2011). However, cells that were derived from this MM-1a/PFDN5 mouse model accumulated more poly-ubiquitinated proteins and were more sensitive to proteasome inhibition. Superficially, no defect in the prefoldin complex was observed, but amino acid substitution in PFDN5 changed the structure of the subunit and altered its affinity for substrates (Abe et al., 2013). These findings demonstrated that the prefoldin complex is an important component of the protein quality control system and performs functions beyond simply acting as a co-translational holdase of newly synthesized polypeptides of cytoskeletal proteins. However, the mechanistic role of the prefoldin complex with regard to the proteasome remained unclear.

Revealing the extended landscape of prefoldin substrates in eukaryotes could shed light on the function of prefoldin during stress conditions, such as limited availability of the protein degradation system. To identify factors that promote the proteasomal degradation of misfolded proteins in yeast, the function of Pfd4 was characterized (Comyn et al., 2016). In the absence of PFD4, the model protein Guk1-7, which is thermally unstable and is a substrate of the proteasome, were partially protected from degradation. The inhibition of Guk1-7 turnover occurred independently of functionality of the proteasome complex and its ubiquitination levels. Importantly, $\mathrm{Pfd} 4$ was shown to interact with the mutant form of Guk1 but not the wildtype version. Thus, Pfd4 acts as a holdase for Guk1-7 to ensure its solubility. In the absence of PFD4, Guk1-7 localized in distinctive foci that were also positive for the aggregation markers Hsp42 and Hsp104. Other thermosensitive alleles were also tested. Different prefoldin subunits stabilized various model proteins to different extents. This indicates that each prefoldin subunit has differential affinity for their substrates (Comyn et al., 2016). This work opens the field for further exploration of a wider 
range of substrates. Much more work is needed to decipher interactions between single prefoldin subunits and specific substrates and substrate classes. Moreover, remaining unclear are whether the function of this holdase depends on a functional prefoldin complex or whether individual subunits act independently from their incorporation in the complex.

The involvement of the prefoldin complex and individual prefoldin subunits in protein quality control seems convincing, but unclear are how its function changes from being a canonical co-translational holdase to a translation-independent chaperone with holdase and disaggregase activity and whether this noncanonical function is independent of TRiC/CCT. Inhibition of the proteasome results in lower levels of amino acids, which in turn activates the integrated stress response. This universal response to unfavorable environmental conditions results in the attenuation of global translation by phosphorylation of eukaryotic initiation factor $2 \alpha$ (eIF2 $\alpha$ ) (Suraweera et al., 2012). Under such cellular conditions, co-translational chaperones might be free to perform other cellular tasks. Such a scenario was described for the ribosome-associated NAC and RAC-Ssb chaperone systems (Olzscha et al., 2011; von Plehwe et al., 2009; Wang et al., 2009).

\section{Implication in the Amelioration of Disease-Associated Protein Aggregation}

Given the emerging role of prefoldin as a factor in maintaining protein solubility under physiological and cellular stress conditions, consistent observations have been made in model systems in which prefoldin can protect from toxic aggregates that are linked to human diseases.

The knockdown of PFDN2 in undifferentiated neuronal cells increased the formation of aggregates of polyQ stretches and polyQ-expanded huntingtin (HTT), gene defects that are related to the neurodegenerative Huntington's disease (Tashiro et al., 2013). The knockdown of PFDN5 also affected aggregation but to a much lesser extent. Importantly, only the aggregation of pathogenic forms was enhanced and not healthy forms. The knockdown of PFDN2 or PFDN5 did not influence the expression of other chaperones, such as HSP70, HSC70, HSP40, and CCTa, suggesting that prefoldin could be a limiting factor to prevent aggregate formation. The overexpression of prefoldin reduced the aggregated form of pathogenic huntingtin. In vitro single-molecule observation by total internal reflection fluorescence microscopy showed that prefoldin suppressed the dimer-to-tetramer stage of HTT aggregation. This indicates that prefoldin inhibits the elongation stage of large oligomers of pathogenic HTT rather than inclusion formation per se (Tashiro et al., 2013).

Similar to HTT aggregation, the formation of a-synuclein aggregates increased upon the knockdown of prefoldin expression in a neuronal cell culture model (Takano et al., 2014). Moreover, ubiquitinated forms of fluorescently tagged wildtype and mutant a-synuclein co-localized with prefoldin (Figure 4B). This observation is consistent with the finding that prefoldin co-localizes with poly-ubiquitinated protein species (Abe et al., 2013). The co-localization of a-synuclein with prefoldin was detected in the lysosome, an organelle that can mediate $\alpha$-synuclein clearance under normal conditions but also under conditions of high protein burden (Mak et al., 2010). Notably, only a small percentage of prefoldin was detected in the lysosome, whereas most remained in the cytoplasm. One unanswered question is whether the effect of prefoldin knockdown on a-synuclein aggregation and toxicity is a direct effect or a consequence of exhaustion of overall folding capacity of the cell. In either case, this work demonstrates that prefoldin might assist with the transfer of misfolded proteins to the autophagy-dependent degradation pathway (Takano et al., 2014) (Figure 4B).

The effect of prefoldin on the oligomerization of amyloid- $\beta$ $(A \beta)$ peptides was also investigated. The fibrillation of $A \beta$ was inhibited in the presence of archaeal prefoldin (Sakono et al., 2008). A similar observation was made in an in vitro model (Sorgjerd et al., 2013). Deposits of $A \beta$ are found in the brain cortex and hippocampus. Prefoldin was detected in both brain regions, but its expression was elevated in a mouse model that exhibited an increase in $A \beta$ deposition. Amyloid- $\beta$ cytotoxicity was reduced in a cell culture model when $A \beta$ oligomers were formed in the presence of human prefoldin. However, the addition of recombinant human prefoldin did not prevent the toxic effect of preformed toxic $A \beta$ oligomers lending further weight to the hypothesis that prefoldin acts on pre-oligomeric misfolded species (Sorgjerd et al., 2013).

In contrast to higher prefoldin expression in brain regions that exhibit an increase in $A \beta$ deposition, a mouse model that expressed a mutant version of Tau exhibited a decrease in PFDN5 expression, which was age-dependent (Kadoyama et al., 2019).

Little is known about prefoldin function during biological aging. However, considering accumulating evidence that prefoldin function can have beneficial effects on the aggregation and toxicity of proteins that are linked to ageassociated diseases, further efforts need to be made to elucidate the potential role of prefoldin during aging. The nematode Caenorhabditis elegans was proven to be a useful model organism for understanding the biology of aging. In $C$. elegans, prefoldin is essential in early development of the embryo because of the need for cytoskeletal structures (Lundin et al., 2008). The use of RNA interference (RNAi) technology allows the knockdown of genes in C. elegans. RNAi against prefoldin subunits is lethal when applied throughout development of the worm. RNAi targeting at later stages of development allows the observation of phenotypes in adult worms. The knockdown of $p f d-6$ did not alter lifespan per se, but $p f d-6$ was partially necessary for the long-lived phenotype of a daf-2 mutant of the insulin/ insulin-like growth factor 1 (IGF-1) pathway (Son et al., 2018). Essentially, this effect was dependent on heat shock factor 1 (HSF-1) and forkhead box O (FOXO/DAF-16) transcription factors, which mediate lifespan extension in daf-2 mutant animals. Moreover, the action of HSF-1 increased the expression of PFD-6 protein levels, possibly by activating the expression of $h s p-70$ and $h s p-90$, which in turn bind and stabilize PFD-6. PFD-6 expression in the daf-2 mutant was necessary for the upregulation of a subset of DAF-16 target genes in daf-2 mutants. The role of PFD-6-dependent lifespan extension in daf- 


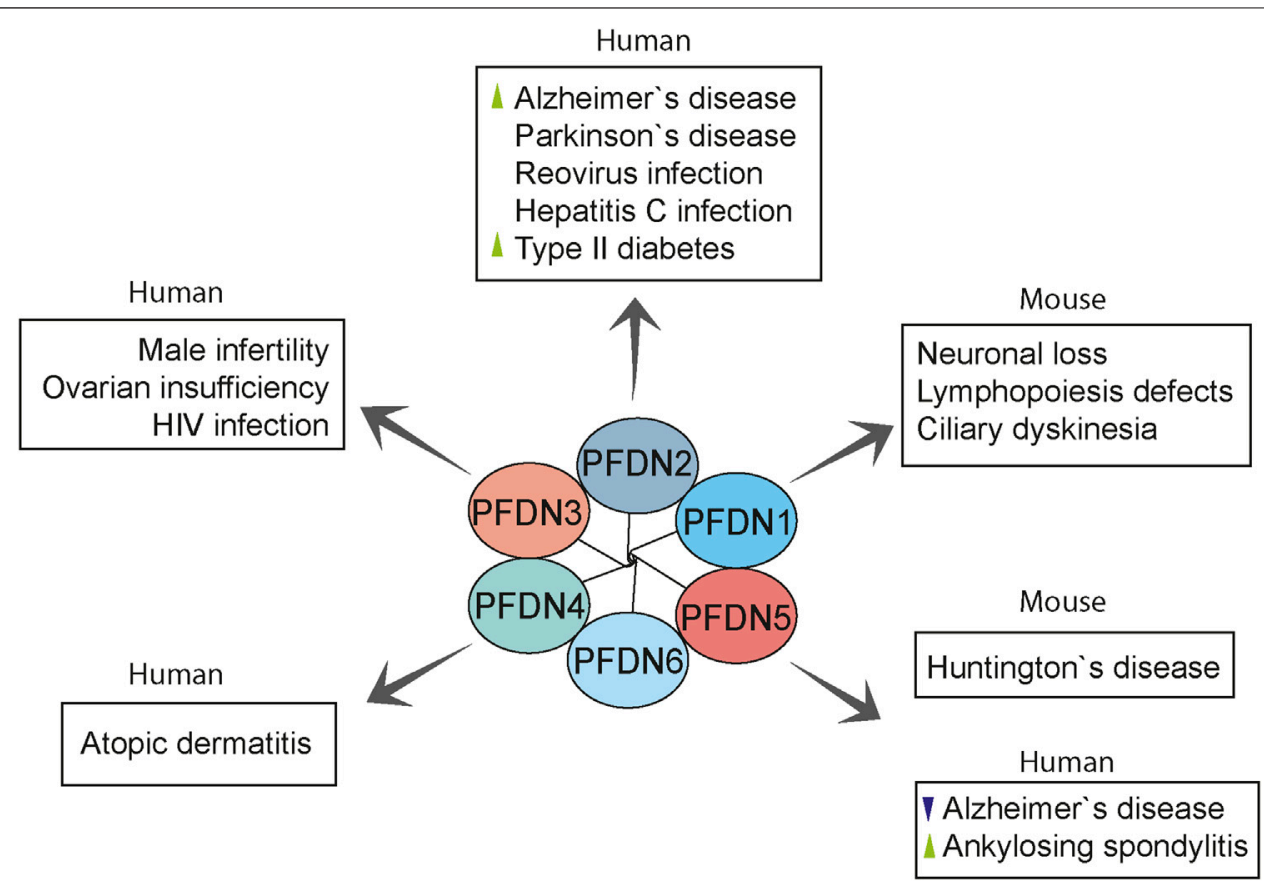

FIGURE 5 | Overview of prefoldin-mediated diseases in humans and mouse models. Prefoldin subunits are involved in various pathologies other than cancer. PFDN2 and PFDN5 were linked with neurodegenerative diseases. PFDN2 and PFDN3 mediate some virus infections. Moreover, prefoldin subunits have distinct roles in the progression of rare anomalies. Changes in prefoldin subunit regulation in human diseases are indicated with arrowhead (green, upregulation; violet, downregulation).

TABLE 2 | Altered expression of prefoldin subunits in human cancer.

\begin{tabular}{|c|c|c|c|}
\hline Subunit & Pathogenic alteration & Type of cancer & References \\
\hline All subunits & Upregulation & Gastric cancer & Yesseyeva et al. (2020) \\
\hline \multirow[t]{4}{*}{ PFDN1 } & Upregulation & Gastric cancer & Yesseyeva et al. (2020), Zhou et al. (2020) \\
\hline & Upregulation & Lung cancer & Wang et al. (2017) \\
\hline & Upregulation & Non-small-cell lung cancer & Peñate et al. (2020) \\
\hline & Upregulation & Colorectal cancers & Wang P et al. (2015); Kwon et al. (2021) \\
\hline \multirow[t]{5}{*}{ PFDN2 } & Upregulation (gain of copy number) & Urothelial carcinoma & López et al. (2013), Riester et al. (2014) \\
\hline & & Bladder cancer & López et al. (2013) \\
\hline & & Gastric cancer & Yesseyeva et al. (2020) \\
\hline & Genetic alterations & Breast tumors & Nami and Wang, (2018) \\
\hline & $\begin{array}{l}\text { Gain or loss of interaction with pathogenic or } \\
\text { altered cellular proteins }\end{array}$ & Liver cancer & $\begin{array}{l}\text { Chang et al., (2006); Dash et al., (2020); El-Serag, } \\
\text { (2012); Farooq et al., (2013) }\end{array}$ \\
\hline $\begin{array}{l}\text { PFDN3 in intact PFDN } \\
\text { complex }\end{array}$ & Downregulation & $\begin{array}{l}\text { Von Hippel-Lindau syndrome (various } \\
\text { types of cancers) }\end{array}$ & $\begin{array}{l}\text { Chesnel et al., (2020); Kim et al., (2018); Tsuchiya } \\
\text { et al., (1996) }\end{array}$ \\
\hline \multirow[t]{4}{*}{ PFDN4 } & Upregulation & Gastric cancer & Feng et al. (2009) \\
\hline & Upregulation & Breast cancer & Collins et al. (2001) \\
\hline & Upregulation & Hepatocellular carcinoma & Wang D et al., (2015) \\
\hline & Downregulation & Colorectal cancer & Miyoshi et al. (2010) \\
\hline \multirow[t]{4}{*}{ PFDN5 } & Downregulation & $\begin{array}{l}\text { Human leukemia, lymphoma, and } \\
\text { tongue cancer }\end{array}$ & Fujioka et al. (2001) \\
\hline & Upregulation of spliced variants & Thyroid neoplasms & Guimarães et al. (2006) \\
\hline & Upregulation & Non-small cell lung cancer & Peñate et al. (2020) \\
\hline & Downregulation & Parathyroid hyperplasia & Santamaría et al. (2005) \\
\hline PFDN6 & Downregulation & Pediatric acute lymphoblastic leukemia & Dehghan-Nayeri et al. (2017) \\
\hline
\end{tabular}


2 mutants is likely related to its non-canonical function in the R2TP (Rvb1-Rvb2-Tah1-Pih1)/prefoldin-like complex rather than the canonical prefoldin complex (Son et al., 2018). This single study in C. elegans demonstrates that we are only in the incipient stages of understanding prefoldin function within a stress-loaded milieu, such as during aging. Much more work needs to be dedicated to unravelling stress-dependent functions of prefoldin.

\section{IMPLICATIONS OF PREFOLDIN IN PATHOLOGICAL CONDITIONS}

Cytoskeletal proteins, such as actin and tubulin, are fundamental to some cellular processes, such as signal transduction, macromolecular trafficking, cell migration, and cell division (Small et al., 1999). Given the role of the prefoldin complex in the biogenesis of cytoskeletal proteins and its additional roles in the cell nucleus (Millan-Zambrano and Chavez, 2014), the prefoldin complex has been speculated to contribute to various disorders. The abnormal expression and function of the prefoldin complex are linked with various disorders, including neurodegenerative diseases, cancer, and viral infections (Figure 5 and Table 2).

\section{Neurodegenerative Diseases}

Neurodegenerative diseases are characterized by the progressive dysfunction of specific neurons and loss of their connections, mainly through the formation of long-insoluble protein fibrils and aggregates (Vaquer-Alicea and Diamond, 2019). Alzheimer's disease is a major neurodegenerative disease that is characterized by progressive memory failure and age-related dementia, which are mainly caused by the aggregation of $A \beta$ protein and formation of amyloid plaques in brain cells (Guo et al., 2020). Autopsies of human brain tissues and cDNA microarray analysis revealed the upregulation of PFDN2 transcript in Alzheimer's disease brains compared with controls (Loring et al., 2001). The association between the prefoldin complex and Alzheimer's disease was also confirmed by single-nucleotide polymorphism analysis and pathway assays (Broer et al., 2011). Interestingly, a study analyzing human whole blood mRNA expression data revealed downregulation of PFDN5 in samples of Alzheimer's patients, which could suggest a link to increased toxicity of $A \beta$ (Tao et al., 2020). The prefoldin complex has also been shown to exert a protective effect against the pathogenesis of Huntington's disease, a progressive neurodegenerative disorder, among a large family of proteopathies. Repeats of the CAG codon (which encodes glutamine) that are greater than 40 in exon 1 of the huntingtin gene elevate the risk of Huntington's disease at age 65 (Langbehn et al., 2004; Langbehn et al., 2019). The extension of polyglutamine regions alters the length and oligomerization of pathogenic huntingtin that subsequently form $\beta$-sheet-rich fibrils and large toxic aggregates (Davies et al., 1997; DiFiglia et al., 1997). Unexpectedly, transgenic mice that carried an integrated human HTT gene with 300 CAG repeats (Q300) had a prolonged lifespan and longer disease onset compared with their parental mice with shorter
CAG repeats (Q150). Transcription studies revealed that phenotype amelioration in Q300 mice was associated with the overexpression of $P f d n 5$ and other genes that are involved in protein folding and localization (Tang et al., 2011). These genes could be therapeutic targets for improving symptoms of Huntington's disease.

PFDN2 has been suggested to be involved in the pathogenesis of the most common neurodegenerative disease, Parkinson's disease. miR-153-3 p microRNA downregulated $\alpha$-synuclein, one protein that is associated with Parkinson's disease, and decreased the abundance of PFDN2 in neuronal SH-SY5Y cells (Patil et al., 2015), suggesting a possible link between Parkinson's disease and the prefoldin complex.

\section{Cancer}

Cancer refers to a group of disorders that are caused by the uncontrolled division of cells that may start in every organ of the body and, in severe cases, spread to other organs (Hausman, 2019). Several studies reported the contribution of the prefoldin complex and its subunits to the onset and progression of some cancers (Table 2) (Liang et al., 2020; Herranz-Montoya et al., 2021). Higher prefoldin expression was observed in cancer cell lines compared with non-cancerous cells (Boudiaf-Benmammar et al., 2013). Additionally, all prefoldin subunits were found to be involved in the development of gastric cancer (Yesseyeva et al. 2020).

The role of cytoskeleton remodeling in cancer metastasis has been confirmed by numerous studies (Aseervatham, 2020). Therefore, one can speculate that prefoldin mediates cancer progression through its role in cytoskeletal proteostasis. Much evidence confirmed that high PFDN1 expression is associated with the metastasis and poor prognosis of gastric cancer, lung cancer, and colorectal cancer (Wang P. et al., 2015; Wang et al., 2017; Zhou et al., 2020; Kwon et al., 2021). High levels of PFDN1 activates the epithelial-mesenchymal transition (EMT), which is necessary for the initiation of metastasis. The mechanism by which PFDN1 initiates activation of the EMT is different in various tumors. For example, in gastric cancer cells, PFDN1 activates alterations of the EMT through $\mathrm{Wnt} / \beta$-catenin signaling (Zhou et al., 2020). In lung tumors, PFDN1 contributes to growth and metastasis by suppressing the transcription of cyclin A. PFDN1 directly interacts with the cyclin A promoter at the transcription initiation site and inhibits cyclin A expression, which eventually activates the EMT and facilitates the migration of lung cancer cells (Wang et al., 2017). A study of PFDN1 mRNA and protein levels in clinical specimens from patients with colorectal cancer showed higher levels of PFDN1 in colorectal cancer samples, particularly the invasive form of the disease, compared with healthy tissues. PFDN1 promotes the proliferation and metastasis of colorectal cancer by maintaining cytoskeletal proteins, particularly F-actin and $\alpha$-tubulin (Wang P. et al., 2015). Clinical specimens of nonsmall cell lung cancer revealed that the overexpression of PFDN1, PFDN3, and PFDN5 are significantly associated with higher mortality and metastasis in non-small cell lung cancer (Peñate et al., 2020). In contrast, microarray data revealed that PFDN1 had stable expression levels in nasopharyngeal carcinoma 
samples, making it a suitable reference gene for expression studies (Guo et al., 2010).

PFDN1 functions as a tumor promoter, and its inhibition has a favorable effect in controlling the progression of some cancers. PFDN1 has been proposed as a target for the treatment of lung cancer (Wang et al., 2017). The silencing of PFDN1 decreased the expression of downstream proteins of $\mathrm{Wnt} / \beta$-catenin signaling, suggesting that PFDN1 may be a specific target to attenuate the development of gastric cancer (Zhou et al., 2020). PFDN1 may also be a diagnostic biomarker and potential candidate for the treatment of colorectal cancer (Wang P. et al., 2015).

PFDN2 is located on chromosome 1q23.3, and increases in its copy number are associated with a poor prognosis and treatment outcome of urothelial carcinoma (López et al., 2013; Riester et al., 2014). The amplification and overexpression of PFDN2 were detected in high-grade tumors of the bladder. Additionally, high levels of PFDN2 were detected in urinary specimens from bladder cancer patients, suggesting that it may be a biomarker for prognosis, tumor staging, and clinical outcome (López et al., 2013). PFDN2 has been shown to interact with most $\alpha$-tubulin and $\beta$-tubulin isoforms, and its genetic alterations are detected in $20 \%$ of breast tumors (Nami and Wang, 2018). Several studies indicated an association between PFDN2 and liver cancer. The alteration of one actin isoform, $\kappa$-actin, was discovered in some hepatocellular carcinoma tissues, with a decrease in its interaction with PFDN2. $\kappa$-Actin rearranges the actin cytoskeleton by replacing $\beta$-actin and becoming a dominant component of the actin cytoskeleton in cancerous hepatoma tissues (Chang et al., 2006). Interactions between $\mathrm{F}$ protein in hepatitis $\mathrm{C}$ virus with PFDN2 promote chronic infection (Tsao et al., 2006), which is known as a predominant risk factor for hepatocellular carcinoma (El-Serag, 2012; Dash et al., 2020). Additionally, alterations of histone deacetylase 1 protein expression were found in hepatocellular carcinoma, and this protein was shown to interact with prefoldin subunits in HepG2 human liver cancer cells (Farooq et al., 2013).

Low levels of the prefoldin complex, particularly the PFDN3 subunit, are associated with the severe phenotype of von HippelLindau (VHL) syndrome (Chesnel et al., 2020). von HippelLindau syndrome is a dominant cancer syndrome that predisposes to various familial or sporadic neoplasms of the central nervous system, adrenal glands, pancreas, retina, and kidney, including clear cell renal cell carcinoma. VHL syndrome is reviewed elsewhere (Varshney et al., 2017). von Hippel-Lindau syndrome is caused by mutations of the VHL tumor suppressor gene and the subsequent loss of its native protein product, $\mathrm{pVHL}$. Tsuchiya and colleagues initially isolated PFDN3, also called von Hippel-Lindau binding protein 1 (VBP1), as a C-terminal binding partner of pVHL (Tsuchiya et al., 1996). PFDN3 was shown to stabilize pVHL and contribute to the pVHL-mediated degradation of hypoxia-inducible factor-1a, ultimately leading to the suppression of cancer metastasis (Kim et al., 2018). Although the main focus of the study by Kim and colleagues was on the PFDN3 subunit, recent research confirmed that the whole prefoldin complex is required to protect pVHL against aggregation. Lower levels of PFDN3 reduce stabilization of the whole prefoldin complex, eventually leading to the poor survival of clear cell renal cell carcinoma patients who harbor VHL missense mutations (Chesnel et al., 2020).

PFDN4, also known as protein C-1, was initially described as a transcription factor that regulates the cell cycle (Iijima et al., 1996). The expression of PFDN4 is regulated by TWIST transcription factor, which plays an important role in the tumorigenesis and metastasis of gastric cancer. The silencing of TWIST increased PFDN4 gene expression levels in gastric cancer cell lines, indicating that PFDN4 might be related to gastric cancer (Feng et al., 2009). Accumulating evidence shows that the genetic locus of PFDN4 confers potential susceptibility to breast cancer. The PFDN4 gene is located on chromosome 20q13.2. A human genome sequence analysis of the $1.2 \mathrm{Mb}$ amplified region of chromosome 20q13.2 showed that the PFDN4 gene is located in the breast cancer amplicon. Amplification of this chromosome region increased PFDN4 expression, suggesting that PFDN4 is an oncogene that is associated with the development of breast cancer (Collins et al., 2001). However, quantitative polymerase chain reaction analysis of archival tissue samples from 54 patients with invasive ductal carcinoma of the breast did not show the significant overexpression of PFDN4 in breast tumors (Born et al., 2005). These disparate results indicate the need for further studies to elucidate the role of PFDN4 in the development of breast cancer.

The gain of whole chromosome $20 \mathrm{q}$ or specific regions therein also plays a role in progressive hepatocellular carcinoma. Analyses of copy number variations of chromosome 20q in hepatocellular carcinoma tumor samples and expression studies revealed that 20q13.12-13.33 gain correlates with the invasiveness of hepatocellular carcinoma. Moreover, PFDN4 is among the genes whose higher expression is associated with a greater risk of extrahepatic metastasis and poor survival (Wang D. et al., 2015). Surgical specimens from 129 patients with colorectal cancer were assessed by Miyoshi et al. (2010) to determine PFDN4 expression and its correlation with clinicopathological futures of colorectal cancer. Higher PFDN4 expression was associated with better survival in colorectal cancer patients. This suggests that in the context of colorectal cancer, PFDN4 functions as a tumor suppressor and may serve as a marker of favorable prognosis (Miyoshi et al., 2010). In breast and liver cancers, high PFDN4 expression promoted carcinogenesis, suggesting that it is an oncogene (Collins et al., 2001; Wang D. et al., 2015). These findings indicate that the impact of PFDN4 in the development of various cancers is tissue-specific.

PFDN5, also known as Myc modulator 1 (Mm-1), was shown to inhibit the transcriptional activity of c-MYC through various mechanisms (Mori et al., 1998; Yoshida et al., 2008). PFDN5 has been suggested to have a tumor-suppressing function. PFDN5 deficiency was shown to be associated with leukemia, lymphoma, and tongue cancer in humans and mammary cancer in canines (Fujioka et al., 2001; Hennecke et al., 2015).

The results of a cDNA microarray analysis of clinical colorectal cancer samples suggested that PFDN5 may be a useful tumor histology and prognosis marker (Tsunoda et al., 2003). The high expression of an alternative splicing variant of PFDN5 was detected in malignant thyroid neoplasms compared 

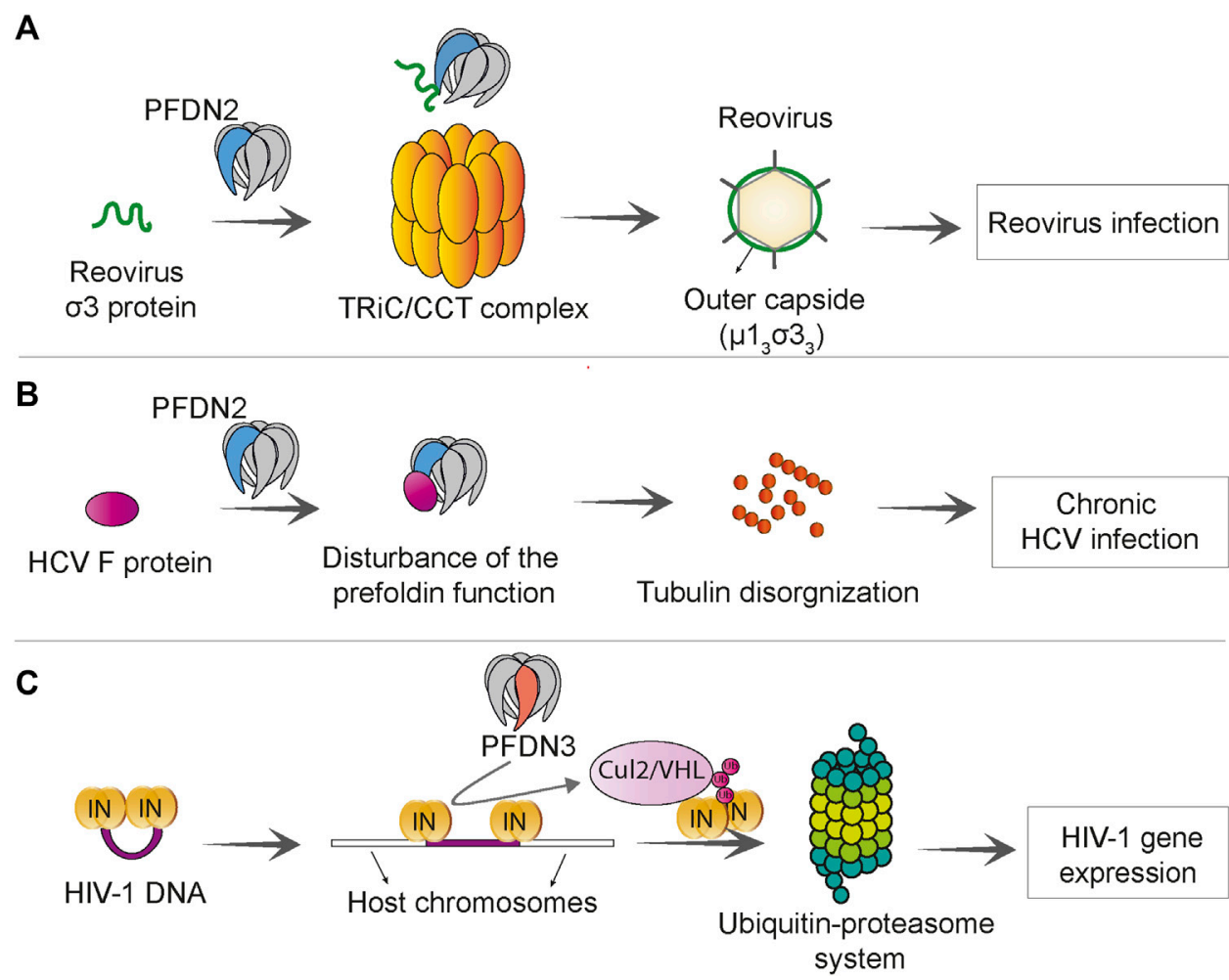

FIGURE 6|Prefoldin is linked to viral infections. (A) PFDN2 subunit of the prefoldin complex interacts with reovirus $\sigma 3$ protein and increases the efficiency of TRiC/ CCT -mediated $\mu 1_{3} \sigma 3_{3}$ assembly, which promotes reovirus infection. (B) The Hepatitis C virus (HCV) F protein interacts with PFDN2 and disturbs the normal function of prefoldin complex in microtubule organization. This leads to persistence of virus in chronic HCV infection. (C) PFDN3 specifically interacts with HIV-1 integrase (IN) and mediates its interaction with cullin 2NHL (Cul2NHL) ubiquitin ligase, which subsequently leads to integrase "polyubiquitination" and proteasome-dependent degradation. This process promotes HIV-1 gene expression at a post-integration step.

with benign lesions and normal thyroid tissues (Guimarães et al., 2006). Moreover, low PFDN5 expression was found to correlate with advanced parathyroid hyperplasia (Santamaría et al., 2005).

The low protein and mRNA expression of PFDN6 was reported to be associated with resistance to the chemotherapy drug dexamethasone in pediatric acute lymphoblastic leukemia. Additionally, low mRNA levels of PFDN6 were detected in bone marrow samples from high-risk cases of pediatric acute lymphoblastic leukemia compared with the low risk and control healthy groups, suggesting that PFDN6 may be a predictive and treatment biomarker of pediatric acute lymphoblastic leukemia (Dehghan-Nayeri et al., 2017).

\section{Viral Infections}

Prefoldin subunits were found to promote some viral infections. The co-chaperone function of the prefoldin complex can be utilized to accelerate the folding of reovirus capsid protein (Knowlton et al., 2021). Reoviruses are rarely pathogenic in early human life but are associated with infection of the respiratory system (Jackson and Muldoon, 1973) and central nervous system (Tyler, 1998), gastroenteritis (Giordano et al., 2002), and celiac disease (Bouziat et al., 2017). The prefoldin complex was shown to augment the TRiC-mediated folding of $\sigma 3$ reovirus capsid protein and enhance efficiency of $\sigma 3 / \mu 1$ assembly to form a heterohexameric proteinic capsid $\left(\mu 1_{3} \sigma 3_{3}\right)$. In this process, PFDN2 directly interacts with reovirus $\sigma 3$ protein (Knowlton et al., 2021) (Figure 6A). Frameshift protein (F protein) of hepatitis $\mathrm{C}$ virus has been shown to bind to PFDN2 and impair function of the prefoldin complex. The F protein-PFDN2 interaction disturbs organization of the tubulin cytoskeleton, subsequently leading to the attenuation of hepatitis $\mathrm{C}$ virus replication and onset of chronic infection (Tsao et al., 2006) (Figure 6B).

PFDN3 has been shown to play a role in human immunodeficiency virus (HIV) infection. HIV-1 viral integrase is required for the integration of proviral DNA into the host genome, but after integration it remains bound to proviral DNA. PFDN3 was shown to bind to the viral integrase (Rain et al., 2009) and mediate its degradation through the ubiquitin-proteasome pathway, which facilitates the transcription of a viral gene. The prefoldin complex and cullin2/VHL ubiquitin ligase are required for appropriate expression of the HIV-1 gene and its postintegration replication (Mousnier et al., 2007) (Figure 6C).

\section{Autoimmune Response and Other Diseases} Clinical research has focused on the implications of prefoldin in tumor progression and as a diagnostic biomarker, but other medical conditions have also been related to prefoldin. Results 
of a mouse study showed that the loss of PFDN1 caused severe phenotypes that were related to cytoskeletal deficiencies, such as lymphopoiesis defects, neuronal loss, and ciliary dyskinesia (Cao et al., 2008). High plasma levels of PFDN2 antibody were reported in patients with type 2 diabetes, especially young adults, suggesting that it may be a clinical biomarker for this disease (Chang et al., 2017). PFDN3 is known to control the level of MutS homologue 4 (MSH4) protein by promoting its poly-ubiquitination in mitotic cells and subsequent degradation (Xu and Her, 2013). MSH4 is essential for meiotic recombination and proper gametogenesis. Lower expression levels of MSH4 were reported to be linked with human male infertility and primary ovarian insufficiency in women (Carlosama et al., 2017; Tang et al., 2020). A genome-wide association study found that the PFDN4 gene was a novel disease-susceptibility locus for atopic dermatitis, an inflammatory skin disorder, in the Japanese population (Hirota et al., 2012). To identify a diagnostic biomarker for uveitis in ankylosing spondylitis, an inflammatory disorder that usually affects the axial spine, Kwon et al. found higher serum levels of anti-PFDN5 antibody in ankylosing spondylitis patients with uveitis. The upregulation of PFDN5 was also confirmed in ocular lesions in a mouse model of this disorder, which was associated with high serum levels of anti-PFDN5 autoantibody. In uveitis, PFDN5 upregulation might be involved in protecting retinal cells against cell death. These observations indicate that serum levels of antiPFDN5 antibody could be a predictive marker of the development of uveitis in ankylosing spondylitis patients (Kwon et al., 2019).

The PFD6 subunit of Plasmodium falciparum (Pf), a eukaryotic parasite that causes malaria, promotes its pathogenesis. Merozoite surface protein-1 (MSP-1) of Pf plays an essential role in the binding of merozoites to human erythrocytes and infecting erythrocytes. Pf PFD6 was shown to interact with MSP-1 suggesting that Pf PFD6 might stabilize and assist the trafficking of MSP-1, which indirectly promotes erythrocyte invasion and pathogenicity of malaria (Kumar et al., 2020).

\section{PREFOLDIN AS A PROMISING CANDIDATE IN NANOMEDICINE}

Several studies investigated the ability of the prefoldin complex to serve as a nanorobot for the intelligent delivery of nano cargo, such as nano drugs. The special structure of prefoldin, which consists of six long coiled-coil tentacles with a flexible central cavity, and the fact that it does not need to utilize ATP to capture and deliver substrates make it an attractive candidate for designing a nano actuator. By introducing amino acid substitutions, a novel prefoldin nano actuator (PNA) was designed that could manipulate hydrophobic nano cargo and inhibit the formation of pathogenic $A \beta$ oligomers. The latter ability suggests that the designed PNA can be effective for the treatment of Alzheimer's disease (Sacks et al., 2018). The binding and release of nano cargo to the PNA cavity can be controlled by $\mathrm{pH}$ and temperature. High temperature decreases the area of the central cavity without affecting the overall conformation of prefoldin. The central cavity of PNA is able to capture a positively charged nano cargo at neutral $\mathrm{pH}$ and release it at acidic pH (Ghaffari et al., 2012; Shokuhfar et al., 2012). Given the higher temperature and lower $\mathrm{pH}$ of the tumor microenvironment compared with normal tissues (Zhang W. et al., 2020), utilization of the PNA may be a site-specific therapeutic strategy whereby an anti-tumor drug is released only at the tumor site while other normal tissues remain unaffected.

\section{PERSPECTIVES}

The molecular co-chaperone prefoldin is a multifaceted protein complex, but the molecular pathways to which it is linked remain poorly described. The diverse involvement of prefoldin in various interconnected cellular functions, ranging from transcriptional regulation to co-translational holdase and post-translational degradation processes, hamper rapid progress in this research field. One future research direction will be to elucidate the cellular and environmental conditions that allow the prefoldin complex to switch between its various functions. Understanding the involvement and regulation of prefoldin under both acute and chronic stress conditions requires further effort. Accumulating evidence suggests that specific prefoldin subunits have functions that are separate from the canonical complex. It is unclear how the balance between formation of the complex and functions of single subunits is regulated. Proteomics approaches that compare landscapes of prefoldin subunit interactions with each other could shed light on independent subunit functions. Pleiotropic positive and negative genetic interactions that are revealed in large-scale screens, mostly in yeast, suggest the existence of extended prefoldin interactors. More efforts should be made to determine whether prefoldin can fulfil its functions without its downstream chaperone TRiC/CCT. Other chaperones could also function together with prefoldin or direct the role of prefoldin under certain cellular conditions.

Finally, the prefoldin complex and its subunits appear to be promising for the discovery of biomarkers for human diseases, including various cancers. More basic research is needed to understand the cause of alterations of prefoldin regulation under pathological conditions to verify whether prefoldin can be a target for treating these diseases.

\section{AUTHOR CONTRIBUTIONS}

IT, SSG, and UT wrote and edited the manuscript. IT and SSG prepared the figures and tables. All authors read and approved the final manuscript.

\section{FUNDING}

This research was funded by the National Science Centre (Grant No. 2018/31/B/NZ1/02401). 


\section{REFERENCES}

Abe, A., Takahashi-Niki, K., Takekoshi, Y., Shimizu, T., Kitaura, H., Maita, H., et al. (2013). Prefoldin Plays a Role as a Clearance Factor in Preventing Proteasome Inhibitor-Induced Protein Aggregation. J. Biol. Chem. 288, 27764-27776. doi:10.1074/jbc.m113.476358

Aikawa, Y., Kida, H., Nishitani, Y., and Miki, K. (2015). Expression, Purification, Crystallization and X-ray Diffraction Studies of the Molecular Chaperone Prefoldin fromHomo Sapiens. Acta Cryst. Sect F 71, 1189-1193. doi:10.1107/s2053230x15013990

Amorim, A. F., Pinto, D., Kuras, L., and Fernandes, L. (2017). Absence of Gim Proteins, but Not GimC Complex, Alters Stress-Induced Transcription. Biochim. Biophys. Acta (Bba) - Gene Regul. Mech. 1860, 773-781. doi:10.1016/j.bbagrm.2017.04.005

Andréasson, C., Ott, M., and Büttner, S. (2019). Mitochondria Orchestrate Proteostatic and Metabolic Stress Responses. EMBO Rep. 20, e47865. doi:10.15252/embr.201947865

Arranz, R., Martín-Benito, J., and Valpuesta, J. M. (2018). Structure and Function of the Cochaperone Prefoldin. Adv. Exp. Med. Biol. 1106, 119-131. doi:10.1007/ 978-3-030-00737-9_9

Aseervatham, J. (2020). Cytoskeletal Remodeling in Cancer. Biology (Basel) 9. doi:10.3390/biology9110385

Balchin, D., Hayer-Hartl, M., and Hartl, F. U. (2016). In Vivo aspects of Protein Folding and Quality Control. Science 353, aac4354. doi:10.1126/science.aac4354

Banks, C. A. S., Miah, S., Adams, M. K., Eubanks, C. G., Thornton, J. L., Florens, L., et al. (2018). Differential HDAC1/2 Network Analysis Reveals a Role for Prefoldin/CCT in HDAC1/2 Complex Assembly. Sci. Rep. 8, 13712. doi:10.1038/s41598-018-32009-w

Blanco-Touriñán, N., Esteve-Bruna, D., and Serrano-Mislata, A., (2021). A Genetic Approach Reveals Different Modes of Action of Prefoldins. Plant Physiol. 187, 1534-1550.

Bogumil, D., Alvarez-Ponce, D., Landan, G., McInerney, J. O., and Dagan, T. (2014). Integration of Two Ancestral Chaperone Systems into One: the Evolution of Eukaryotic Molecular Chaperones in Light of Eukaryogenesis. Mol. Biol. Evol. 31, 410-418. doi:10.1093/molbev/mst212

Born, M., Quintanilla-Fend, L., Braselmann, H., Reich, U., Richter, M., Hutzler, P., et al. (2005). Simultaneous Over-expression of theHer2/neu andPTK6 Tyrosine Kinases in Archival Invasive Ductal Breast Carcinomas. J. Pathol. 205, 592-596. doi:10.1002/path.1720

Boudiaf-Benmammar, C., Cresteil, T., and Melki, R. (2013). The Cytosolic Chaperonin CCT/TRiC and Cancer Cell Proliferation. PLoS One 8, e60895. doi:10.1371/journal.pone.0060895

Bouziat, R., Hinterleitner, R., Brown, J. J., Stencel-Baerenwald, J. E., Ikizler, M., Mayassi, T., et al. (2017). Reovirus Infection Triggers Inflammatory Responses to Dietary Antigens and Development of Celiac Disease. Science 356, 44-50. doi:10.1126/science.aah5298

Broer, L., Ikram, M. A., Schuur, M., DeStefano, A. L., Bis, J. C., Liu, F., et al. (2011). Association of HSP70 and its Co-chaperones with Alzheimer's Disease. Jad 25, 93-102. doi:10.3233/jad-2011-101560

Cao, J. (2016). Analysis of the Prefoldin Gene Family in 14 Plant Species. Front. Plant Sci. 7, 317. doi:10.3389/fpls.2016.00317

Cao, S., Carlesso, G., Osipovich, A. B., Llanes, J., Lin, Q., Hoek, K. L., et al. (2008). Subunit 1 of the Prefoldin Chaperone Complex Is Required for Lymphocyte Development and Function. J. Immunol. 181, 476-484. doi:10.4049/ jimmunol.181.1.476

Carlosama, C., Elzaiat, M., Patiño, L. C., Mateus, H. E., Veitia, R. A., and Laissue, P. (2017). A Homozygous Donor Splice-Site Mutation in the Meiotic Gene MSH4 Causes Primary Ovarian Insufficiency. Hum. Mol. Genet. 26, 3161-3166. doi:10.1093/hmg/ddx199

Chang, D. C., Piaggi, P., Hanson, R. L., Knowler, W. C., Bogardus, C., and Krakoff, J. (2017). Autoantibodies against PFDN2 Are Associated with an Increased Risk of Type 2 Diabetes: A Case-Control Study. Diabetes Metab. Res. Rev. 33. doi:10.1002/dmrr.2922

Chang, K., Yang, P., Lai, H., Yeh, T., Chen, T., and Yeh, C. (2006). Identification of a Novel Actin Isoform in Hepatocellular Carcinoma. Hepatol. Res. 36, 33-39. doi:10.1016/j.hepres.2006.05.003
Chaudhari, S. N., and Kipreos, E. T. (2018). The Energy Maintenance Theory of Aging: Maintaining Energy Metabolism to Allow Longevity. Bioessays 40, e1800005. doi:10.1002/bies.201800005

Chen, Y., Li, Y., Peng, Y., Zheng, X., Fan, S., Yi, Y., et al. (2018). $\Delta$ Np63a DownRegulates C-Myc Modulator MM1 via E3 Ligase HERC3 in the Regulation of Cell Senescence. Cell Death Differ 25, 2118-2129. doi:10.1038/s41418-0180132-5

Chesnel, F., Couturier, A., Alusse, A., Gagné, J.-P., Poirier, G. G., Jean, D., et al. (2020). The prefoldin complex stabilizes the von Hippel-Lindau protein against aggregation and degradation. Plos Genet. 16, e1009183. doi:10.1371/ journal.pgen.1009183

Chintalapudi, S. R., and Jablonski, M. M. (2017). Systems Genetics Analysis to Identify the Genetic Modulation of a Glaucoma-Associated Gene. Methods Mol. Biol. 1488, 391-417. doi:10.1007/978-1-4939-6427-7_18

Collins, C., Volik, S., and Kowbel, D., (2001). Comprehensive Genome Sequence Analysis of a Breast Cancer Amplicon. Genome Res. 11, 1034-1042. doi:10.1101/gr.gr1743r

Comyn, S. A., Young, B. P., Loewen, C. J., and Mayor, T. (2016). Prefoldin Promotes Proteasomal Degradation of Cytosolic Proteins with Missense Mutations by Maintaining Substrate Solubility. Plos Genet. 12, e1006184. doi:10.1371/journal.pgen.1006184

Cooper, G. M. (2000). The Cell: A Molecular Approach. 2nd edition. Sunderland, Massachusetts: Sinauer Associates.

Costanzo, M., VanderSluis, B., Koch, E. N., Baryshnikova, A., Pons, C., Tan, G., et al. (2016). A Global Genetic Interaction Network Maps a Wiring Diagram of Cellular Function. Science 353. aaf1420. doi:10.1126/science.aaf1420

Dash, S., Aydin, Y., Widmer, K. E., and Nayak, L. (2020). Hepatocellular Carcinoma Mechanisms Associated with Chronic HCV Infection and the Impact of Direct-Acting Antiviral Treatment. Jhc Vol. 7, 45-76. doi:10.2147/ jhc.s221187

Dehghan-Nayeri, N., Rezaei-Tavirani, M., Omrani, M. D., Gharehbaghian, A., Goudarzi Pour, K., and Eshghi, P. (2017). Identification of Potential Predictive Markers of Dexamethasone Resistance in Childhood Acute Lymphoblastic Leukemia. J. Cel Commun. Signal. 11, 137-145. doi:10.1007/s12079-016-0357-3

El-Serag, H. B. (2012). Epidemiology of Viral Hepatitis and Hepatocellular Carcinoma. Gastroenterology 142, 1264-1273. e1261. doi:10.1053/ j.gastro.2011.12.061

Esteve-Bruna, D., Carrasco-López, C., Blanco-Touriñán, N., Iserte, J., CallejaCabrera, J., Perea-Resa, C., et al. (2020). Prefoldins Contribute to Maintaining the Levels of the Spliceosome LSM2-8 Complex through Hsp90 in Arabidopsis. Nucleic Acids Res. 48, 6280-6293. doi:10.1093/nar/gkaa354

Fandrich, M., Tito, M. A., Leroux, M. R., Rostom, A. A., Hartl, F. U., Dobson, C. M., et al. (2000). Observation of the Noncovalent Assembly and Disassembly Pathways of the Chaperone Complex MtGimC by Mass Spectrometry. Proc. Natl. Acad. Sci. 97, 14151-14155. doi:10.1073/pnas.240326597

Fang, Y., Wang, X., Yang, D., Lu, Y., Wei, G., Yu, W., et al. (2021). Relieving Cellular Energy Stress in Aging, Neurodegenerative, and Metabolic Diseases, SIRT1 as a Therapeutic and Promising Node. Front. Aging Neurosci. 13, 738686. doi:10.3389/fnagi.2021.738686

Farooq, M., Hozzein, W. N., Elsayed, E. A., Taha, N. A., and Wadaan, M. A. M. (2013). Identification of Histone Deacetylase 1 Protein Complexes in Liver Cancer Cells. Asian Pac. J. Cancer Prev. 14, 915-921. doi:10.7314/ apjcp.2013.14.2.915

Feng, M.-Y., Wang, K., Shi, Q.-T., Yu, X.-W., and Geng, J.-S. (2009). Gene Expression Profiling in TWIST-Depleted Gastric Cancer Cells. Anat. Rec. 292, 262-270. doi:10.1002/ar.20802

Friesen, H., Humphries, C., Ho, Y., Schub, O., Colwill, K., and Andrews, B. (2006). Characterization of the Yeast Amphiphysins Rvs161p and Rvs167p Reveals Roles for the Rvs Heterodimer In Vivo. MBoC 17, 1306-1321. doi:10.1091/ mbc.e05-06-0476

Fujioka, Y., Taira, T., Maeda, Y., Tanaka, S., Nishihara, H., Iguchi-Ariga, S. M. M., et al. (2001). MM-1, a C-Myc-Binding Protein, Is a Candidate for a Tumor Suppressor in Leukemia/lymphoma and Tongue Cancer. J. Biol. Chem. 276, 45137-45144. doi:10.1074/jbc.m106127200

Geissler, S., Siegers, K., and Schiebel, E. (1998). A Novel Protein Complex Promoting Formation of Functional Alpha - and Gamma -tubulin. EMBO J. 17, 952-966. doi:10.1093/emboj/17.4.952 
Gestaut, D., Roh, S. H., Ma, B., Pintilie, G., Joachimiak, L. A., Leitner, A., et al. (2019). The Chaperonin TRiC/CCT Associates with Prefoldin through a Conserved Electrostatic Interface Essential for Cellular Proteostasis. Cell 177, 751-765. e715. doi:10.1016/j.cell.2019.03.012

Ghaffari, A., Shokuhfar, A., and Ghasemi, R. H. (2012). Capturing and Releasing a Nano Cargo by Prefoldin Nano Actuator. Sensors Actuators B: Chem. 171-172, 1199-1206. doi:10.1016/j.snb.2012.06.077

Giordano, M. O., Martínez, L. C., Isa, M. B., Ferreyra, L. J., Canna, F., Paván, J. V., et al. (2002). Twenty Year Study of the Occurrence of Reovirus Infection in Hospitalized Children with Acute Gastroenteritis in Argentina. Pediatr. Infect. Dis. J. 21, 880-882. doi:10.1097/00006454-200209000-00021

Glover, D. J., and Clark, D. S. (2015). Oligomeric Assembly Is Required for Chaperone Activity of the Filamentous $\gamma$-prefoldin. FEBS J. 282, 2985-2997. doi:10.1111/febs.13341

Goehring, A. S., Mitchell, D. A., Tong, A. H. Y., Keniry, M. E., Boone, C., and Sprague, G. F. (2003). Synthetic Lethal Analysis Implicates Ste20p, a P21Activated Protein Kinase, in Polarisome Activation. MBoC 14, 1501-1516. doi:10.1091/mbc.e02-06-0348

Goldman, D. H., Kaiser, C. M., Milin, A., Righini, M., Tinoco, I., and Bustamante, C. (2015). Mechanical Force Releases Nascent Chain-Mediated Ribosome Arrest In Vitro and In Vivo. Science 348, 457-460. doi:10.1126/science.1261909

Guimarães, G. S., Latini, F. R., and Camacho, C. P. (2006). Identification of Candidates for Tumor-specific Alternative Splicing in the Thyroid. Genes Chromosomes Cancer 45, 540-553.

Guo, T., Zhang, D., Zeng, Y., Huang, T. Y., Xu, H., and Zhao, Y. (2020). Molecular and Cellular Mechanisms Underlying the Pathogenesis of Alzheimer's Disease. Mol. Neurodegeneration 15, 40. doi:10.1186/s13024-020-00391-7

Guo, Y., Chen, J.-x., Yang, S., Fu, X.-p., Zhang, Z., Chen, K.-h., et al. (2010). Selection of Reliable Reference Genes for Gene Expression Study in Nasopharyngeal Carcinoma. Acta Pharmacol. Sin 31, 1487-1494. doi:10.1038/aps.2010.115

Hagio, Y., Kimura, Y., Taira, T., Fujioka, Y., Iguchi-Ariga, S. M. M., and Ariga, H. (2006). Distinct Localizations and Repression Activities of MM-1 Isoforms toward C-Myc. J. Cel. Biochem. 97, 145-155. doi:10.1002/jcb.20619

Han, A., Li, J., Li, Y., Wang, Y., Bergholz, J., Zhang, Y., et al. (2016). p63a Modulates C-Myc Activity via Direct Interaction and Regulation of MM1 Protein Stability. Oncotarget 7, 44277-44287. doi:10.18632/oncotarget.10187

Hansen, W. J., Cowan, N. J., and Welch, W. J. (1999). Prefoldin-nascent Chain Complexes in the Folding of Cytoskeletal Proteins. J. Cel Biol 145, 265-277. doi:10.1083/jcb.145.2.265

Hartl, F. U., and Hayer-Hartl, M. (2002). Molecular Chaperones in the Cytosol: from Nascent Chain to Folded Protein. Science 295, 1852-1858. doi:10.1126/ science. 1068408

Hausman, D. M. (2019). What Is Cancer? Perspect. Biol. Med. 62, 778-784. doi:10.1353/pbm.2019.0046

Hennecke, S., Beck, J., Bornemann-Kolatzki, K., Neumann, S., Escobar, H. M., Nolte, I., et al. (2015). Prevalence of the Prefoldin Subunit 5 Gene Deletion in Canine Mammary Tumors. PLoS One 10, e0131280. doi:10.1371/ journal.pone.0131280

Herranz-Montoya, I., Park, S., and Djouder, N. (2021). A Comprehensive Analysis of Prefoldins and Their Implication in Cancer. iScience 24, 103273. doi:10.1016/ j.isci.2021.103273

Hirota, T., Takahashi, A., Kubo, M., Tsunoda, T., Tomita, K., Sakashita, M., et al. (2012). Genome-wide Association Study Identifies Eight New Susceptibility Loci for Atopic Dermatitis in the Japanese Population. Nat. Genet. 44, 1222-1226. doi:10.1038/ng.2438

Iijima, M., Kano, Y., Nohno, T., and Namba, M. (1996). Cloning of cDNA with Possible Transcription Factor Activity at the G1-S Phase Transition in Human Fibroblast Cell Lines. Acta Med. Okayama 50, 73-77. doi:10.18926/AMO/ 30489

Jackson, G. G., and Muldoon, R. L. (1973). Viruses Causing Common Respiratory Infection in Man. IV. Reoviruses and Adenoviruses. J. Infect. Dis. 128, 811-866. doi:10.1093/infdis/128.6.811

Jones, R. D., and Gardner, R. G. (2016). Protein Quality Control in the Nucleus. Curr. Opin. Cel Biol. 40, 81-89. doi:10.1016/j.ceb.2016.03.002

Kabir, M. A., Uddin, W., Narayanan, A., Reddy, P. K., Jairajpuri, M. A., Sherman, F., et al. (20112011). Functional Subunits of Eukaryotic Chaperonin CCT/TRiC in Protein Folding. J. Amino Acids 2011, 843206. doi:10.4061/2011/843206
Kadoyama, K., Matsuura, K., Takano, M., Maekura, K., Inoue, Y., and Matsuyama, S. (2019). Changes in the Expression of Prefoldin Subunit 5 Depending on Synaptic Plasticity in the Mouse hippocampus. Neurosci. Lett. 712, 134484. doi:10.1016/j.neulet.2019.134484

Kim, J. A., Choi, D. K., Min, J. S., Kang, I., Kim, J. C., Kim, S., et al. (2018). VBP 1 Represses Cancer Metastasis by Enhancing HIF - 1a Degradation Induced by pVHL. Febs j 285, 115-126. doi:10.1111/febs.14322

Knowlton, J. J., Gestaut, D., Ma, B., Taylor, G., Seven, A. B., Leitner, A., et al. (2021). Structural and Functional Dissection of Reovirus Capsid Folding and Assembly by the Prefoldin-TRiC/CCT Chaperone Network. Proc. Natl. Acad. Sci. U S A. 118, e2018127118. doi:10.1073/pnas.2018127118

Krogan, N. J., Kim, M., Tong, A., Golshani, A., Cagney, G., Canadien, V., et al. (2003). Methylation of Histone H3 by Set2 in Saccharomyces cerevisiae Is Linked to Transcriptional Elongation by RNA Polymerase II. Mol. Cel Biol 23, 4207-4218. doi:10.1128/mcb.23.12.4207-4218.2003

Kumar, V., Rumaishaand Behl, A. (2020). Prefoldin Subunit 6 of Plasmodium Falciparum Binds Merozoite Surface Protein-1 (MSP-1). FEBS Open Bio 3. doi:10.1002/2211-5463.13022

Kwon, M., Rubio, G., Nolan, N., Auteri, P., Volmar, J. A., Adem, A., et al. (2021). FILIP1L Loss Is a Driver of Aggressive Mucinous Colorectal Adenocarcinoma and Mediates Cytokinesis Defects through PFDN1. Cancer Res. 81, 5523-5539. doi:10.1158/0008-5472.can-21-0897

Kwon, O. C., Lee, E.-J., Lee, J. Y., Youn, J., Kim, T.-H., Hong, S., et al. (2019). Prefoldin 5 and Anti-prefoldin 5 Antibodies as Biomarkers for Uveitis in Ankylosing Spondylitis. Front. Immunol. 10, 384 . doi:10.3389/ fimmu.2019.00384

Langbehn, D., Brinkman, R., Falush, D., Paulsen, J., and Hayden, M. (2004). A New Model for Prediction of the Age of Onset and Penetrance for Huntington's Disease Based on CAG Length. Clin. Genet. 65, 267-277. doi:10.1111/j.13990004.2004.00241.x

Langbehn, D. R., Stout, J. C., Gregory, S., Mills, J. A., Durr, A., Leavitt, B. R., et al. (2019). Association of CAG Repeats with Long-Term Progression in Huntington Disease. JAMA Neurol. 76, 1375-1385. doi:10.1001/ jamaneurol.2019.2368

Lee, Y., Smith, R. S., Jordan, W., King, B. L., Won, J., Valpuesta, J. M., et al. (2011). Prefoldin 5 Is Required for normal Sensory and Neuronal Development in a Murine Model. J. Biol. Chem. 286, 726-736. doi:10.1074/jbc.m110.177352

Leroux, M. R., Fandrich, M., and Klunker, D. (1999). MtGimC, a Novel Archaeal Chaperone Related to the Eukaryotic Chaperonin Cofactor GimC/prefoldin. EMBO J. 18, 6730-6743. doi:10.1093/emboj/18.23.6730

Liang, J., Xia, L., Oyang, L., Lin, J., Tan, S., Yi, P., et al. (2020). The Functions and Mechanisms of Prefoldin Complex and Prefoldin-Subunits. Cell Biosci 10, 87. doi:10.1186/s13578-020-00446-8

Liu, H., Liu, X., Jin, H., Yang, F., Gu, W., and Li, X. (2013). Proteomic Analysis of Knock-Down HLA-G in Invasion of Human Trophoblast Cell Line JEG-3. Int. J. Clin. Exp. Pathol. 6, 2451-2459.

Locascio, A., Blázquez, M. A., and Alabadí, D. (2013). Dynamic Regulation of Cortical Microtubule Organization through Prefoldin-DELLA Interaction. Curr. Biol. 23, 804-809. doi:10.1016/j.cub.2013.03.053

López, V., González-Peramato, P., and Suela, J., (2013). Identification of Prefoldin Amplification (1q23.3-q24.1) in Bladder Cancer Using Comparative Genomic Hybridization (CGH) Arrays of Urinary DNA. J. Transl Med. 11, 182.

Loring, J. F., Wen, X., Lee, J. M., Seilhamer, J., and Somogyi, R. (2001). A Gene Expression Profile of Alzheimer's Disease. DNA Cel Biol. 20, 683-695. doi:10.1089/10445490152717541

Lundin, V. F., Srayko, M., Hyman, A. A., and Leroux, M. R. (2008). Efficient Chaperone-Mediated Tubulin Biogenesis Is Essential for Cell Division and Cell Migration in C. elegans. Develop. Biol. 313, 320-334. doi:10.1016/ j.ydbio.2007.10.022

Ma, H.-C., Lin, T.-W., Li, H., Iguchi-Ariga, S. M. M., Ariga, H., Chuang, Y.-L., et al. (2008). Hepatitis C Virus ARFP/F Protein Interacts with Cellular MM-1 Protein and Enhances the Gene Trans-activation Activity of C-Myc. J. Biomed. Sci. 15, 417-425. doi:10.1007/s11373-008-9248-9

Mak, S. K., McCormack, A. L., Manning-Boğ, A. B., Cuervo, A. M., and Di Monte, D. A. (2010). Lysosomal Degradation of a-Synuclein In Vivo. J. Biol. Chem. 285, 13621-13629. doi:10.1074/jbc.m109.074617

Marracci, S., Martini, D., Giannaccini, M., Giudetti, G., Dente, L., and Andreazzoli, M. (2015). Comparative Expression Analysis of Pfdn6a and Tcp1? during 
Xenopus Development. Int. J. Dev. Biol. 59, 235-240. doi:10.1387/ ijdb.140275ma

Martin-Benito, J., Boskovic, J., and Gomez-Puertas, P., (2002). Structure of Eukaryotic Prefoldin and of its Complexes with Unfolded Actin and the Cytosolic Chaperonin CCT. EMBO J. 21, 6377-6386. doi:10.1093/emboj/ cdf640

Millán-Zambrano, G., and Chávez, S. (2014). Nuclear Functions of Prefoldin. Open Biol. 4. doi:10.1098/rsob.140085

Millán-Zambrano, G., Rodríguez-Gil, A., Peñate, X., de Miguel-Jiménez, L., Morillo-Huesca, M., Krogan, N., et al. (2013). The Prefoldin Complex Regulates Chromatin Dynamics during Transcription Elongation. Plos Genet. 9, e1003776. doi:10.1371/journal.pgen.1003776

Ming Sun, S., Batté, A., Elmer, M., van der Horst, S. C., van Welsem, T., Bean, G., et al. (2020). A Genetic Interaction Map Centered on Cohesin Reveals Auxiliary Factors Involved in Sister Chromatid Cohesion in S. cerevisiae. J. Cel Sci 133. doi: $10.1242 /$ jcs. 237628

Mitchell, L., Lambert, J.-P., Gerdes, M., Al-Madhoun, A. S., Skerjanc, I. S., Figeys, D., et al. (2008). Functional Dissection of the NuA4 Histone Acetyltransferase Reveals its Role as a Genetic Hub and that Eaf1 Is Essential for Complex Integrity. Mol. Cel Biol 28, 2244-2256. doi:10.1128/mcb.01653-07

Miyoshi, N., Ishii, H., Mimori, K., Nishida, N., Tokuoka, M., Akita, H., et al. (2010). Abnormal Expression of PFDN4 in Colorectal Cancer: a Novel Marker for Prognosis. Ann. Surg. Oncol. 17, 3030-3036. doi:10.1245/s10434-010-1138-5

Mori, K., Maeda, Y., Kitaura, H., Taira, T., Iguchi-Ariga, S. M. M., and Ariga, H. (1998). MM-1, a Novel C-Myc-Associating Protein that Represses Transcriptional Activity of C-Myc. J. Biol. Chem. 273, 29794-29800. doi:10.1074/jbc.273.45.29794

Mousnier, A., Kubat, N., Massias-Simon, A., Segeral, E., Rain, J.-C., Benarous, R., et al. (2007). von Hippel Lindau binding protein 1-mediated degradation of integrase affects HIV-1 gene expression at a postintegration step. Proc. Natl. Acad. Sci. 104, 13615-13620. doi:10.1073/pnas.0705162104

Nami, B., and Wang, Z. (2018). Genetics and Expression Profile of the Tubulin Gene Superfamily in Breast Cancer Subtypes and its Relation to Taxane Resistance, 10.274 doi:10.3390/cancers10080274Cancers (Basel)

Narita, R., Kitaura, H., Torii, A., Tashiro, E., Miyazawa, M., Ariga, H., et al. (2012). Rabring7 Degrades C-Myc through Complex Formation with MM-1. PLoS One 7, e41891. doi:10.1371/journal.pone.0041891

Ohtaki, A., Kida, H., Miyata, Y., Ide, N., Yonezawa, A., Arakawa, T., et al. (2008). Structure and Molecular Dynamics Simulation of Archaeal Prefoldin: the Molecular Mechanism for Binding and Recognition of Nonnative Substrate Proteins. J. Mol. Biol. 376, 1130-1141. doi:10.1016/j.jmb.2007.12.010

Olzscha, H., Schermann, S. M., Woerner, A. C., Pinkert, S., Hecht, M. H., Tartaglia, G. G., et al. (2011). Amyloid-like Aggregates Sequester Numerous Metastable Proteins with Essential Cellular Functions. Cell 144, 67-78. doi:10.1016/ j.cell.2010.11.050

Palumbo, V., Pellacani, C., Heesom, K. J., Rogala, K. B., Deane, C. M., MottierPavie, V., et al. (2015). Misato Controls Mitotic Microtubule Generation by Stabilizing the TCP-1 Tubulin Chaperone Complex. Curr. Biol. 25, 1777-1783. doi:10.1016/j.cub.2015.05.033

Pan, X., Reissman, S., Douglas, N. R., Huang, Z., Yuan, D. S., Wang, X., et al. (2010). Trivalent Arsenic Inhibits the Functions of Chaperonin Complex. Genetics 186, 725-734. doi:10.1534/genetics.110.117655

Patil, K. S., Basak, I., Pal, R., Ho, H.-P., Alves, G., Chang, E. J., et al. (2015). A Proteomics Approach to Investigate miR-153-3p and miR-205-5p Targets in Neuroblastoma Cells. PLoS One 10, e0143969. doi:10.1371/journal.pone.0143969

Payán-Bravo, L., Fontalva, S., and Peñate, X., (2021). Human Prefoldin Modulates Co-transcriptional Pre-mRNA Splicing. Nucleic Acids Res. 49, 6267-6280.

Peñate, X., Praena-Fernández, J.M, and Romero Pareja, P. (2020). Overexpression of Canonical Prefoldin Associates with the Risk of Mortality and Metastasis in Non-Small Cell Lung Cancer.Cancers (Basel), 12 (4):1052. doi:10.3390/ cancers 12041052

Povarova, O. I., Uversky, V. N., Kuznetsova, I. M., and Turoverov, K. K. (2014). Actinous enigma or Enigmatic Actin. Intrinsically Disordered Proteins 2, e34500. doi:10.4161/idp.34500

Rain, J.-C., Cribier, A., Gérard, A., Emiliani, S., and Benarous, R. (2009). Yeast Two-Hybrid Detection of Integrase-Host Factor Interactions. Methods 47, 291-297. doi:10.1016/j.ymeth.2009.02.002
Riester, M., Werner, L., Bellmunt, J., Selvarajah, S., Guancial, E. A., Weir, B. A., et al. (2014). Integrative Analysis of 1q23.3 Copy-Number Gain in Metastatic Urothelial Carcinoma. Clin. Cancer Res. 20, 1873-1883. doi:10.1158/10780432.ccr-13-0759

Rothman, S. (2010). How Is the Balance between Protein Synthesis and Degradation Achieved? Theor. Biol. Med. Model. 7, 25. doi:10.1186/17424682-7-25

Russmann, F., Stemp, M. J., Monkemeyer, L., Etchells, S. A., Bracher, A., and Hartl, F. U. (2012). Folding of Large Multidomain Proteins by Partial Encapsulation in the Chaperonin TRiC/CCT. Proc. Natl. Acad. Sci. 109, 21208-21215. doi:10.1073/pnas.1218836109

Sacks, D., Sacks, D., Baxter, B., Campbell, B. C. V., Carpenter, J. S., Cognard, C., et al. (2018). Multisociety Consensus Quality Improvement Revised Consensus Statement for Endovascular Therapy of Acute Ischemic Stroke. AJNR Am. J. Neuroradiol 39, E61-E632. doi:10.3174/ajnr.A5638

Sahlan, M., Zako, T., and Yohda, M. (2018). Prefoldin, a Jellyfish-like Molecular Chaperone: Functional Cooperation with a Group II Chaperonin and beyond. Biophys. Rev. 10, 339-345. doi:10.1007/s12551-018-0400-0

Saibil, H. (2013). Chaperone Machines for Protein Folding, Unfolding and Disaggregation. Nat. Rev. Mol. Cel Biol 14, 630-642. doi:10.1038/nrm3658

Sakono, M., Zako, T., Ueda, H., Yohda, M., and Maeda, M. (2008). Formation of Highly Toxic Soluble Amyloid Beta Oligomers by the Molecular Chaperone Prefoldin. FEBS J. 275, 5982-5993. doi:10.1111/j.1742-4658.2008.06727.x

Santamaría, I., Alvarez-Hernández, D., and Jofré, R. (2005). Progression of Secondary Hyperparathyroidism Involves Deregulation of Genes Related to DNA and RNA Stability. Kidney Int. 67, 2267-2279. doi:10.1111/j.15231755.2005.00330

Satou, A., Taira, T., Iguchi-Ariga, S. M. M., and Ariga, H. (2001). A Novel Transrepression Pathway of C-Myc. J. Biol. Chem. 276, 46562-46567. doi:10.1074/jbc.m104937200

Shokuhfar, A., Ghaffari, A., and Ghasemi, R. H. (2012). Cavity Control of Prefoldin Nano Actuator (PNA) by Temperature and pH. Nano-micro Lett. 4, 110-117. doi:10.1007/bf03353701

Siegert, R., Leroux, M. R., Scheufler, C., Hartl, F. U., and Moarefi, I. (2000). Structure of the Molecular Chaperone Prefoldin. Cell 103, 621-632. doi:10.1016/s0092-8674(00)00165-3

Simons, C. T., Staes, A., Rommelaere, H., Ampe, C., Lewis, S. A., and Cowan, N. J. (2004). Selective Contribution of Eukaryotic Prefoldin Subunits to Actin and Tubulin Binding. J. Biol. Chem. 279, 4196-4203. doi:10.1074/jbc.m306053200

Small, J. V., Rottner, K., and Kaverina, I. (1999). Functional Design in the Actin Cytoskeleton. Curr. Opin. Cel Biol. 11, 54-60. doi:10.1016/s0955-0674(99) 80007-6

Son, H. G., Seo, K., Seo, M., Park, S., Ham, S., An, S. W. A., et al. (2018). Prefoldin 6 Mediates Longevity Response from Heat Shock Factor 1 to FOXO in C. elegans. Genes Dev. 32, 1562-1575. doi:10.1101/gad.317362.118

Sörgjerd, K. M., Zako, T., Sakono, M., Stirling, P. C., Leroux, M. R., Saito, T., et al. (2013). Human Prefoldin Inhibits Amyloid- $\beta$ (A $\beta$ ) Fibrillation and Contributes to Formation of Nontoxic A $\beta$ Aggregates. Biochemistry 52, 3532-3542. doi:10.1021/bi301705c

Suraweera, A., Münch, C., Hanssum, A., and Bertolotti, A. (2012). Failure of Amino Acid Homeostasis Causes Cell Death Following Proteasome Inhibition. Mol. Cel 48, 242-253. doi:10.1016/j.molcel.2012.08.003

Takano, M., Tashiro, E., Kitamura, A., Maita, H., Iguchi-Ariga, S. M. M., Kinjo, M., et al. (2014). Prefoldin Prevents Aggregation of a-synuclein. Brain Res. 1542, 186-194. doi:10.1016/j.brainres.2013.10.034

Tang, B., Seredenina, T., Coppola, G., Kuhn, A., Geschwind, D. H., Luthi-Carter, R., et al. (2011). Gene Expression Profiling of R6/2 Transgenic Mice with Different CAG Repeat Lengths Reveals Genes Associated with Disease Onset and Progression in Huntington's Disease. Neurobiol. Dis. 42, 459-467. doi:10.1016/j.nbd.2011.02.008

Tang, D., Xu, C., Geng, H., Gao, Y., Cheng, H., Ni, X., et al. (2020). A Novel Homozygous Mutation in the Meiotic Gene MSH4 Leading to Male Infertility Due to Non-obstructive Azoospermia. Am. J. Transl Res. 12, 8185-8191.

Tao, Y., Han, Y., Yu, L., Wang, Q., Leng, S. X., and Zhang, H. (2020). The Predicted Key Molecules, Functions, and Pathways that Bridge Mild Cognitive Impairment (MCI) and Alzheimer's Disease (AD). Front. Neurol. 11, 233. doi:10.3389/fneur.2020.00233 
Tashiro, E., Zako, T., Muto, H., Itoo, Y., Sörgjerd, K., Terada, N., et al. (2013). Prefoldin Protects Neuronal Cells from Polyglutamine Toxicity by Preventing Aggregation Formation. J. Biol. Chem. 288, 19958-19972. doi:10.1074/jbc.m113.477984

Thulasiraman, V., Yang, C. F., and Frydman, J. (1999). In Vivo newly Translated Polypeptides Are Sequestered in a Protected Folding Environment. EMBO J. 18, 85-95. doi:10.1093/emboj/18.1.85

Tong, A. H. Y., Lesage, G., Bader, G. D., Ding, H., Xu, H., Xin, X., et al. (2004). Global Mapping of the Yeast Genetic Interaction Network. Science 303, 808-813. doi:10.1126/science.1091317

Tsao, M.-L., Chao, C.-H., and Yeh, C.-T. (2006). Interaction of Hepatitis C Virus F Protein with Prefoldin 2 Perturbs Tubulin Cytoskeleton Organization. Biochem. Biophysical Res. Commun. 348, 271-277. doi:10.1016/j.bbrc.2006.07.062

Tsuchiya, H., Iseda, T., and Hino, O. (1996). Identification of a novel protein (VBP1) binding to the von Hippel-Lindau (VHL) tumor suppressor gene product. Cancer Res. 56, 2881-2885.

Tsunoda, T., Koh, Y., Koizumi, F., Tsukiyama, S., Ueda, H., Taguchi, F., et al. (2003). Differential Gene Expression Profiles and Identification of the Genes Relevant to Clinicopathologic Factors in Colorectal Cancer Selected by cDNA Array Method in Combination with Principal Component Analysis. Int. J. Oncol. 23, 49-59. doi:10.3892/ijo.23.1.49

Tyler, K. L. (1998). Pathogenesis of Reovirus Infections of the central Nervous System. Curr. Top. Microbiol. Immunol. 233, 93-124. doi:10.1007/978-3-642-72095-6_6

Vainberg, I. E., Lewis, S. A., Rommelaere, H., Ampe, C., Vandekerckhove, J., Klein, H. L., et al. (1998). Prefoldin, a Chaperone that Delivers Unfolded Proteins to Cytosolic Chaperonin. Cell 93, 863-873. doi:10.1016/s0092-8674(00)81446-4

Vaquer-Alicea, J., and Diamond, M. I. (2019). Propagation of Protein Aggregation in Neurodegenerative Diseases. Annu. Rev. Biochem. 88, 785-810. doi:10.1146/ annurev-biochem-061516-045049

Varshney, N., Kebede, A. A., Owusu-Dapaah, H., Lather, J., Kaushik, M., and Bhullar, J. S. (2017). A Review of Von Hippel-Lindau Syndrome. jkcvhl 4, 20-29. doi:10.15586/jkcvhl.2017.88

von Plehwe, U., Berndt, U., Conz, C., Chiabudini, M., Fitzke, E., Sickmann, A., et al. (2009). The Hsp70 Homolog Ssb Is Essential for Glucose Sensing via the SNF1 Kinase Network. Genes Dev. 23, 2102-2115. doi:10.1101/gad.529409

Wang, D., Shi, W., Tang, Y., Liu, Y., He, K., Hu, Y., et al. (2017). Prefoldin 1 Promotes EMT and Lung Cancer Progression by Suppressing Cyclin A Expression. Oncogene 36, 885-898. doi:10.1038/onc.2016.257

Wang, D., Zhu, Z.-Z., Jiang, H., Zhu, J., Cong, W.-M., Wen, B.-J., et al. (2015). Multiple Genes Identified as Targets for 20q13.12-13.33 Gain Contributing to Unfavorable Clinical Outcomes in Patients with Hepatocellular Carcinoma. Hepatol. Int. 9, 438-446. doi:10.1007/s12072-015-9642-0

Wang, P., Zhao, J., Yang, X., Guan, S., Feng, H., Han, D., et al. (2015). PFDN1, an Indicator for Colorectal Cancer Prognosis, Enhances Tumor Cell Proliferation and Motility through Cytoskeletal Reorganization. Med. Oncol. 32, 264. doi:10.1007/s12032-015-0710-Z

Wang, Y., Meriin, A. B., Zaarur, N., Romanova, N. V., Chernoff, Y. O., Costello, C. E., et al. (2009). Abnormal Proteins Can Form Aggresome in Yeast: Aggresometargeting Signals and Components of the Machinery. FASEB j. 23, 451-463. doi:10.1096/fj.08-117614

Watanabe, K.-i., Ozaki, T., Nakagawa, T., Miyazaki, K., Takahashi, M., Hosoda, M., et al. (2002). Physical Interaction of P73 with C-Myc and MM1, a C-MycBinding Protein, and Modulation of the P73 Function. J. Biol. Chem. 277, 15113-15123. doi:10.1074/jbc.m111281200

Webster, J. M., Darling, A. L., Uversky, V. N., and Blair, L. J. (2019). Small Heat Shock Proteins, Big Impact on Protein Aggregation in Neurodegenerative Disease. Front. Pharmacol. 10, 1047. doi:10.3389/fphar.2019.01047
Whitehead, T. A., Boonyaratanakornkit, B. B., Hollrigl, V., and Clark, D. S. (2007) A Filamentous Molecular Chaperone of the Prefoldin Family from the DeepSea Hyperthermophile Methanocaldococcus Jannaschii. Protein Sci. 16, 626-634. doi:10.1110/ps.062599907

Xu, Y., and Her, C. (2013). VBP1 Facilitates Proteasome and Autophagy-mediated Degradation of MutS Homologue hMSH4. FASEB j. 27, 4799-4810. doi:10.1096/fj.13-235127

Yam, A. Y., Xia, Y., Lin, H.-T. J., Burlingame, A., Gerstein, M., and Frydman, J. (2008). Defining the TRiC/CCT Interactome Links Chaperonin Function to Stabilization of Newly Made Proteins with Complex Topologies. Nat. Struct. Mol. Biol. 15, 1255-1262. doi:10.1038/nsmb.1515

Yamane, T., Shimizu, T., Takahashi-Niki, K., Takekoshi, Y., Iguchi-Ariga, S. M. M., and Ariga, H. (2015). Deficiency of Spermatogenesis and Reduced Expression of Spermatogenesis-Related Genes in Prefoldin 5-mutant Mice. Biochem. Biophys. Rep. 1, 52-61. doi:10.1016/j.bbrep.2015.03.005

Yesseyeva, G., Aikemu, B., Hong, H., Yu, C., Dong, F., Sun, J., et al. (2020). Prefoldin Subunits (PFDN1-6) Serve as Poor Prognostic Markers in Gastric Cancer. Biosci. Rep. 40. doi:10.1042/BSR20192712

Yoshida, T., Kitaura, H., Hagio, Y., Sato, T., Iguchi-Ariga, S. M. M., and Ariga, H. (2008). Negative Regulation of the Wnt Signal by MM-1 through Inhibiting Expression of the Wnt4 Gene. Exp. Cel Res. 314, 1217-1228. doi:10.1016/ j.yexcr.2008.01.002

Zhang, J., Han, Q., Song, Y., Chen, Q., and Xia, X. (2015). Analysis of Subcellular Prefoldin 1 Redistribution during Rabies Virus Infection. Jundishapur J. Microbiol. 8, e24757. doi:10.5812/jjm.24757v2

Zhang, J., Xie, M., Li, M., Ding, J., Pu, Y., Bryan, A. C., et al. (2020). Overexpression of a Prefoldin $\beta$ Subunit Gene Reduces Biomass Recalcitrance in the Bioenergy Crop Populus. Plant Biotechnol. J. 18, 859-871. doi:10.1111/pbi.13254

Zhang, W., Yu, L., Ji, T., and Wang, C. (2020). Tumor MicroenvironmentResponsive Peptide-Based Supramolecular Drug Delivery System. Front. Chem. 8, 549. doi:10.3389/fchem.2020.00549

Zhang, Y., Rai, M., Wang, C., Gonzalez, C., and Wang, H. (2016). Prefoldin and Pins Synergistically Regulate Asymmetric Division and Suppress Dedifferentiation. Sci. Rep. 6, 23735. doi:10.1038/srep23735

Zhou, C., Guo, Z., Xu, L., Jiang, H., Sun, P., Zhu, X., et al. (2020). PFND1 Predicts Poor Prognosis of Gastric Cancer and Promotes Cell Metastasis by Activating the Wnt/ $\beta$-Catenin Pathway. Ott 13, 3177-3186. doi: $10.2147 /$ ott.s236929

Conflict of Interest: The authors declare that the research was conducted in the absence of any commercial or financial relationships that could be construed as a potential conflict of interest.

Publisher's Note: All claims expressed in this article are solely those of the authors and do not necessarily represent those of their affiliated organizations, or those of the publisher, the editors, and the reviewers. Any product that may be evaluated in this article, or claim that may be made by its manufacturer, is not guaranteed or endorsed by the publisher.

Copyright (C) 2022 Tahmaz, Shahmoradi Ghahe and Topf. This is an open-access article distributed under the terms of the Creative Commons Attribution License (CC $B Y)$. The use, distribution or reproduction in other forums is permitted, provided the original author(s) and the copyright owner(s) are credited and that the original publication in this journal is cited, in accordance with accepted academic practice. No use, distribution or reproduction is permitted which does not comply with these terms. 